\title{
SPATIAL AND TEMPORAL WHITE NOISES UNDER SUBLINEAR G-EXPECTATION
}

\author{
JI XIAOJUN AND PENG SHIGE
}

\begin{abstract}
In the framework of sublinear expectation, we have introduced a new type of $G$-Gaussian random fields, which contain a type of spatial white noise as a special case. Based on this result, we also have introduced a spatial-temporal $G$-white noise. Different from the case of linear expectation, in which the probability measure need to be known, under the uncertainty of the probability measure, the spatial white noises are intrinsically different from the temporal one.
\end{abstract}

Key words. sublinear expectation, $G$-Brownian motion, $G$-Gaussian random field, $G$-white noise, Spatial and temporal white noise.

\section{INTRODUCTION}

The axiomatic formulation of probability space $(\Omega, \mathcal{F}, P)$ is a powerful and elegant mathematical framework for quantitative studies of uncertainties. A typical example is to introduce a Wiener probability measure $P$ on the space of $d$-dimensional continuous paths $\Omega=C\left([0, \infty) ; \mathbb{R}^{d}\right)$, with $\mathcal{F}=\mathcal{B}(\Omega)$. The canonical process $B_{t}(\omega)=\omega_{t}, t \geq 0$, is then a standard Brownian motion, namely, under the Wiener probability measure $P$, the process $B$ becomes incrementally stable, independent and continuous. It turns out that any random vector of the form $\left(B_{t_{1}}, \cdots, B_{t_{n}}\right)$ is normally distributed and thus $B$ is a Gaussian process, and all increments $B_{t+s}-B_{t}$ are independent of $\left(B_{t_{1}}, \cdots, B_{t_{n}}\right)$, for $t_{1}, \cdots, t_{n} \leq t$, and identically distributed with respect to $B_{s}$.

But in most cases of our real world, the probability measure itself is essentially unknown. This type of higher level uncertainty can be described by a family of probability measures $\left\{P_{\theta}\right\}_{\theta \in \Theta}$ such that we are unable to know "true one". In this case the notion of sublinear expectation

$$
\mathbb{E}[X]=\sup _{\theta \in \Theta} \int_{\Omega} X d P_{\theta} .
$$

is often used to obtain a robust expectation. This type of formulation induces a new type of sublinear expectation $\mathbb{E}$ which has been widely investigated, see, e.g., Huber [9](1981), Walley [18](1991). See also the corresponding coherent risk measures representation of Arzner et al [1](1999), Delbaen [4](2002) and Föllmer \& Schied [8](2002).

A very intereting question is that can we follow the idea of Kolmogorov-Wiener, to use the above mentioned idea to construct a basic nonlinear expectation $\mathbb{E}[\cdot]$, under which the same canonical process $B$ becomes a "nonlinear" Brownian motion? Peng [12](2007) introduced a notion of $G$-Brownian motion which is still the same canonical process. But the Wiener probability measure $P$ was replaced by the corresponding sublinear expectation, called G-expectation, such that $B$ becomes a continuous stochastic process 
whose increments are stable and independent. It turns out to be a typical model of stochastic processes for which the uncertainty of probabilities is dynamically and intrinsically taken into account. We can also use this $G$-Brownian motion to generate a very rich class of stochastic processes such as $G$-OU process, geometric $G$-Brownian motions, through the corresponding stochastic differential equations driven by this $G$ Brownian motion, thanks to a generalized Itô's calculus.

But it is worth to point out that, for each $t>0, B_{t}$ is not symmetric with respect to $B_{2 t}-B_{t}$ in the level of distributions. We know that $B_{2 t}-B_{t}$ is independent from $B_{t}$, but in general $B_{t}$ is not independent from $B_{2 t}-B_{t}$. In fact, we can check that, although $B_{2 t}-B_{t}$ and $B_{t}$ are both $G$-normally distributed, but the 2-dimensional random vector $\left(B_{2 t}-B_{t}, B_{t}\right)$ is not $G$-normal. In general $Y$ is independent from $X$ does not imply that $X$ is independent from $Y$. In fact the notion of independence becomes a typical temporal relation. For example, a random sequence $\left\{X_{i}\right\}_{i=1}^{\infty}$ is a i.i.d. sequence under a nonlinear expectation is mainly for the case where $i \in \mathbb{N}$ is a time index, namely $\left\{X_{i}\right\}_{i=1}^{\infty}$ is a time series. How to introduce a space-indexed random fields becomes an interesting and challenging problem.

In this paper, we introduce a new type of spatial-temporal white noise in the presence of probability model uncertainties which is a natural generalization of the classical spatial white noise (see, among others, Walsh [19], Dalang [2], Da Prato and J. Zabczyk [3]). But we emphasis that, in the framework of nonlinear expectation, the spaceindexed increments does not satisfy the property of independence. Once the nonlinear $G$-expectation reduced to its special situation of linear expectation, the property of independence for the space indexed part turns out to be true, thanks for the Fubini Lemma for the linear expectation. The Gaussian property for the time indexed part also turns out to be true, thanks to the important property of (linear) normal distributions that independent Gaussian random variables constitute a Gaussian random vector.

The objective of this paper is to construct a white noise of space-indexed, as well as space-time-indexed. We will see that such type of space-indexed white noise is a special type of Gaussian random field under probability uncertainty, satisfying distributional invariant of rotations and translations (see Proposition 24).

An important and interesting point observed in Peng [17] is that a G-Brownian motion $\left(B_{t}\right)_{t>0}$ is very efficient to quantify time-indexed random fields, but it is no longer suitable to describe space-indexed uncertainties. A new notion of Gaussian process was then introduced in Peng [17]. Our main point of view is that under a sublinear expectation, a framework of $G$-Gaussian random fields is suitable for describing spatial random fields.

In this paper we will systematically develop a new type of spatial Gaussian random fields under a sublinear expectation space. It is remarkable that is a finite distribution of a $\left(B_{t_{1}}, \cdots, B_{t_{n}}\right)$ is no longer $G$-normally distributed.

In many literature, the index $t$ in Brownian motion $B_{t}$ is also treated as a spatial parameter, i.e., $t=x \in \mathbb{R}$. This method is widely used in the study of space-time indexed random field. In fact, this can be justified by Fubini theorem, in which time and space is exchangeable. However, Fubini theorem does not hold for the nonlinear expectation framework. So we need to establish a new theoretical framework in which 
space and time are treated separately for obtaining a spatial-temporal random fields, and then develop a new space-time indexed white noise theory.

We hope that this new framework of spatial-temporal random fields is useful in quantitative financial risk measures, complex random networks, stochastic partial differential equations, non-equlibrium statistical physics, stochastic control system and games, large scale robust economics, and spatial and temporal indexed data.

With the quickly increasing complexity of the internet network, its uncertainty in probability measures as a space indexed random field is increasing. There is an urgent need for an exact theoretical framework of nonlinear expectation in this field.

We establish the general random field under the nonlinear expectation framework. In particular, we construct spatial random fields and spatial-temporal white noises, and then the related stochastic calculus theory. Specially, if the nonlinear expectation degrades to a linear expectation, the corresponding random field theory and space-time indexed white noise coincide with classical results.

This paper is organized as follows. In Section 2, we review some basic notions and results of nonlinear expectation theory, the notion of distributions and independence of random vectors, the notion of $G$-normal distribution, $G$-Brownian motion and their basic properties. In Section 3, we first provide a generalized Kolmogorov's existence theorem based from a consistently defined family of finite dimensional distributions in the framework of nonlinnear expectations. The existence and consistence of a type of $G$-Gaussian random fields is also provided. In Section 4, we construct the spatial white noise and the corresponding stochastic integral. The existence and stochastic integral of space-time-indexed white noise are discussed in Section 5.

We are aware of deep research results of Föllmer [6] and Föllmer \& Klüppelberg [7] (2014) on spatial risk measures and will explore the relations between the two seemingly very different approaches.

\section{Preliminaries}

In this section, we present some basic notions and properties in nonlinear expectation. More details can be found in [5], [12], and [15] .

2.1. Basic notions of nonlinear expectations. Let $\Omega$ be a given nonempty set and $\mathcal{H}$ be a linear space of real-valued functions on $\Omega$ such that if $X_{1}, \ldots, X_{d} \in \mathcal{H}$, then $\varphi\left(X_{1}, X_{2}, \ldots, X_{d}\right) \in \mathcal{H}$ for each $\varphi \in C_{\text {l.Lip }}\left(\mathbb{R}^{d}\right)$, where $C_{\text {l.Lip }}\left(\mathbb{R}^{d}\right)$ denotes the linear space of functions satisfying for each $x, y \in \mathbb{R}^{d}$,

$|\varphi(x)-\varphi(y)| \leq C_{\varphi}\left(1+|x|^{m}+|y|^{m}\right)|x-y|$, for some $C_{\varphi}>0, m \in \mathbb{N}$ depending on $\varphi$.

$\mathcal{H}$ is considered as the space of random variables. $X=\left(X_{1}, \ldots, X_{d}\right), X_{i} \in \mathcal{H}, 1 \leq i \leq d$, is called a $d$-dimensional random vector, denoted by $X \in \mathcal{H}^{d}$.

Definition 1. A sublinear expectation $\hat{\mathbb{E}}$ on $\mathcal{H}$ is a functional $\hat{\mathbb{E}}: \mathcal{H} \rightarrow \mathbb{R}$ satisfying the following properties: for each $X, Y \in \mathcal{H}$,

(i) Monotonicity: $\hat{\mathbb{E}}[X] \geq \hat{\mathbb{E}}[Y]$ if $X \geq Y$;

(ii) Constant preserving: $\hat{\mathbb{E}}[c]=c$ for $c \in \mathbb{R}$;

(iii) Sub-additivity: $\hat{\mathbb{E}}[X+Y] \leq \hat{\mathbb{E}}[X]+\hat{\mathbb{E}}[Y]$; 
(iv) Positive homogeneity: $\hat{\mathbb{E}}[\lambda X]=\lambda \hat{\mathbb{E}}[X]$ for $\lambda \geq 0$. The triplet $(\Omega, \mathcal{H}, \hat{\mathbb{E}})$ is called a sublinear expectation space. If only (i) and (ii) are satisfied, then $\hat{\mathbb{E}}$ is called a nonlinear expectation and the triplet $(\Omega, \mathcal{H}, \hat{\mathbb{E}})$ is called a nonlinear expectation space.

(v) Regularity: If $\left\{X_{i}\right\}_{i=1}^{\infty} \subset \mathcal{H}$ be such that $X_{i}(\omega) \downarrow 0$ as $i \rightarrow \infty$, for each $\omega \in \Omega$, then

$$
\lim _{i \rightarrow \infty} \hat{\mathbb{E}}\left[X_{i}\right]=0 .
$$

In this paper we are mainly interested in sublinear expectations satisfying the regular condition.

Theorem 2. Let $\hat{\mathbb{E}}$ be a sublinear expectation on $(\Omega, \mathcal{H})$ satisfying the regularity condition (v) in Definition 1. Then there exists a family of unique probability measures $\left\{P_{\theta}\right\}_{\theta \in \Theta}$, such that

$$
\hat{\mathbb{E}}[X]=\max _{\theta \in \Theta} \int_{\Omega} X(\omega) d P_{\theta}, \forall X \in \mathcal{H}
$$

Let $(\Omega, \mathcal{H}, \hat{\mathbb{E}})$ be a nonlinear (resp. sublinear) expectation space. For each given $d$-dimensional random vector $X$, we define a functional on $C_{l . L i p}\left(\mathbb{R}^{d}\right)$ by

$$
\mathbb{F}_{X}[\varphi]:=\hat{\mathbb{E}}[\varphi(X)] \text {, for each } \varphi \in C_{l . L i p}\left(\mathbb{R}^{d}\right) \text {. }
$$

It is easy to verify that $\left(\mathbb{R}^{d}, C_{\text {l.Lip }}\left(\mathbb{R}^{d}\right), \mathbb{F}_{X}\right)$ forms a nonlinear (resp. sublinear) expectation space. $\mathbb{F}_{X}$ is called the distribution of $X$. Two $d$-dimensional random vectors $X_{1}$ and $X_{2}$ defined respectively on nonlinear expectation spaces $\left(\Omega_{1}, \mathcal{H}_{1}, \hat{\mathbb{E}}_{1}\right)$ and $\left(\Omega_{2}, \mathcal{H}_{2}, \hat{\mathbb{E}}_{2}\right)$ are called identically distributed, denoted by $X_{1} \stackrel{d}{=} X_{2}$, if $\mathbb{F}_{X_{1}}=\mathbb{F}_{X_{2}}$, i.e.,

$$
\hat{\mathbb{E}}_{1}\left[\varphi\left(X_{1}\right)\right]=\hat{\mathbb{E}}_{2}\left[\varphi\left(X_{2}\right)\right] \text { for each } \varphi \in C_{l . L i p}\left(\mathbb{R}^{d}\right) .
$$

Similar to the classical case, Peng [14](2008) gave the following definition of convergence in distribution.

Definition 3. Let $X_{n}, n \geq 1$, be a sequence of $d$-dimensional random vectors defined respectively on nonlinear (resp. sublinear) expectation spaces $\left(\Omega_{n}, \mathcal{H}_{n}, \hat{\mathbb{E}}_{n}\right)$. $\left\{X_{n}: n \geq\right.$ $1\}$ is said to converge in distribution if, for each fixed $\varphi \in C_{b . L i p}\left(\mathbb{R}^{d}\right),\left\{\mathbb{F}_{X_{n}}[\varphi]: n \geq 1\right\}$ is a Cauchy sequence, where $C_{b . L i p}\left(\mathbb{R}^{d}\right)$ denotes the set of all bounded and Lipschitz functions on $\mathbb{R}^{d}$. Define

$$
\mathbb{F}[\varphi]=\lim _{n \rightarrow \infty} \mathbb{F}_{X_{n}}[\varphi],
$$

then the triplet $\left(\mathbb{R}^{d}, C_{\text {b.Lip }}\left(\mathbb{R}^{d}\right), \mathbb{F}\right)$ forms a nonlinear (resp. sublinear) expectation space.

The following notion of the independence of random variables under a nonlinear expectation is very useful (see Peng [15](2010)).

Definition 4. Let $(\Omega, \mathcal{H}, \hat{\mathbb{E}})$ be a nonlinear expectation space. An $n$-dimensional random vector $Y$ is said to be independent from another $m$-dimensional random vector $X$ under the expectation $\hat{\mathbb{E}}$ if, for each test function $\varphi \in C_{l . L i p}\left(\mathbb{R}^{m+n}\right)$, we have

$$
\hat{\mathbb{E}}[\varphi(X, Y)]=\hat{\mathbb{E}}\left[\hat{\mathbb{E}}[\varphi(x, Y)]_{x=X}\right] .
$$


Let $\bar{X}$ and $X$ be two $m$-dimensional random vectors on $(\Omega, \mathcal{H}, \hat{\mathbb{E}}) . \quad \bar{X}$ is called an independent copy of $X$ if $\bar{X} \stackrel{d}{=} X$ and $\bar{X}$ is independent from $X$.

Remark 5. It is important to note that " $Y$ is independent from $X$ " does not imply that " $X$ is independent from $Y$ " (see Peng [15]).

For each $p \geq 1$, let $\mathbb{L}^{p}(\Omega)$ be the completion of $\mathcal{H}$ under the Banach norm $\|X\|_{\mathbb{L}^{p}}:=$ $\left(\hat{\mathbb{E}}\left[|X|^{p}\right]\right)^{\frac{1}{p}}$. It is easy to verify that $\mathbb{L}^{p}(\Omega) \subset \mathbb{L}^{p^{\prime}}(\Omega)$ for each $1 \leq p^{\prime} \leq p$. Since $|\hat{\mathbb{E}}[X]-\hat{\mathbb{E}}[Y]| \leq \hat{\mathbb{E}}[|X-Y|], \hat{\mathbb{E}}$ can be continuously extended to the mapping from $\mathbb{L}^{1}(\Omega)$ to $\mathbb{R}$ and properties $(i)-(i v)$ of definition 1 still hold. Moreover, $\left(\Omega, \mathbb{L}^{1}(\Omega), \hat{\mathbb{E}}\right)$ also forms a sublinear expectation space, which is called a complete sublinear expectation space.

We say that $X=Y$ in $\mathbb{L}^{p}(\Omega)$ if $\hat{\mathbb{E}}\left[|X-Y|^{p}\right]=0$, and denote by $X \geq Y$ or $Y \leq X$ if $X-Y=(X-Y)^{+}$.

2.2. G-normal distributions under a sublinear expectation space. In the rest part of this paper, we focus ourselves to a sublinear expectation space $(\Omega, \mathcal{H}, \hat{\mathbb{E}})$. The following simple lemma is quite useful:

Lemma 6. (see Peng [15]) If a ramdom variable $\xi \in \mathcal{H}$ satisfies $\hat{\mathbb{E}}[\xi]=\hat{\mathbb{E}}[-\xi]=0$, then we have

$$
\hat{\mathbb{E}}[\xi+\eta]=\hat{\mathbb{E}}[\eta], \quad \forall \eta \in \mathcal{H} .
$$

Definition 7. A $d$-dimensional random vector $X=\left(X_{1}, \cdots, X_{d}\right)$ on a sublinear expectation space $(\Omega, \mathcal{H}, \hat{\mathbb{E}})$ is called (centralized) $G$-normally distributed if

$$
a X+b \bar{X} \stackrel{d}{=} \sqrt{a^{2}+b^{2}} X \quad \text { for } a, b \geq 0,
$$

where $\bar{X}$ is an independent copy of $X$.

We denote by $\mathbb{S}(d)$ the collection of all $d \times d$ symmetric matrices. The distribution of a $G$-normally distributed random vector $X$ defined on $(\Omega, \mathcal{H}, \hat{\mathbb{E}})$ is uniquely characterized by a function $G=G_{X}: \mathbb{S}(d) \mapsto \mathbb{R}$ defined as follows:

$$
G_{X}(Q):=\frac{1}{2} \hat{\mathbb{E}}[\langle Q X, X\rangle], \quad Q \in \mathbb{S}(d) .
$$

It is easy to check that $G$ is a sublinear and continuous function monotone in $Q \in \mathbb{S}(d)$ in the following sense: for each $Q, \bar{Q} \in \mathbb{S}(d)$,

$$
\left\{\begin{array}{l}
G(Q+\bar{Q}) \leq G(Q)+G(\bar{Q}), \\
G(\lambda Q)=\lambda G(Q), \quad \forall \lambda \geq 0 \\
G(Q) \geq G(\bar{Q}), \quad \text { if } Q \geq \bar{Q}
\end{array}\right.
$$

Proposition 8. Let $G: \mathbb{S}(d) \rightarrow \mathbb{R}$ be a given sublinear and continuous function, monotone in $Q \in \mathbb{S}(d)$ in the sense of (2.2). Then there exists a $G$-normally distributed $d$-dimensional random vector $X$ on some sublinear expectation space $(\Omega, \mathcal{H}, \hat{\mathbb{E}})$ satisfying (2.1). Moreover, if $\bar{X}$ is also a normally distributed random variable such that

$$
G_{X}(Q)=G_{\bar{X}}(Q), \quad \text { for any } Q \in \mathbb{S}(d),
$$

then we have $X \stackrel{d}{=} \bar{X}$. 
The function $G_{X}(\cdot)$ associated to the distribution function of $G$-normal variable $X$ is called the generating function of $X$.

Proposition 9. Let $G$ be given as in Proposition 8 and $X$ be a d-dimensional random vector on a sublinear expectation space $(\Omega, \mathcal{H}, \hat{\mathbb{E}})$ such that $X$ is $G$-normally distributed. For each $\varphi \in C_{\text {l.Lip }}\left(\mathbb{R}^{d}\right)$, define

$$
u(t, x):=\hat{\mathbb{E}}[\varphi(x+\sqrt{t} X)],(t, x) \in[0, \infty) \times \mathbb{R}^{d} .
$$

Then $u$ is the unique viscosity solution of the $G$-heat equation

$$
\partial_{t} u-G\left(D^{2} u\right)=0,\left.\quad u\right|_{t=0}=\varphi(x)
$$

The following property of a $G$-normally distributed random vector $\xi$ is easy to check:

Proposition 10. Let $\xi$ be a d-dimensional G-normally distributed random vector characterized by its generating function

$$
G_{\xi}(Q):=\frac{1}{2} \hat{\mathbb{E}}[\langle Q \xi, \xi\rangle], \quad Q \in \mathbb{S}(d) .
$$

Then for any matrix $K \in \mathbb{R}^{m \times d}, K \xi$ is also an $m$-dimensional $G$-normally distributed random vector. Its corresponding generating function is

$$
G_{K \xi}(Q)=\frac{1}{2} \hat{\mathbb{E}}\left[\left\langle K^{T} Q K \xi, \xi\right\rangle\right], \quad Q \in \mathbb{S}(m) .
$$

Proof. Let $\bar{\xi}$ be an independent copy of $\xi$. It is clear that $K \bar{\xi}$ is also an independent copy of $K \xi$. Thus, for each $\varphi \in C_{l \text {.Lip }}\left(\mathbb{R}^{m}\right)$, the function $\varphi_{K}(x):=\varphi(K x), x \in \mathbb{R}^{d}$, is also a $C_{\text {l.Lip }}\left(\mathbb{R}^{d}\right)$ function. It follows from the definition of the $G$-normal distribution that, for any $a, b \geq 0$,

$$
\hat{\mathbb{E}}[\varphi(a K \xi+b K \bar{\xi})]=\hat{\mathbb{E}}\left[\varphi_{K}(a \xi+b \bar{\xi})\right]=\hat{\mathbb{E}}\left[\varphi_{K}\left(\sqrt{a^{2}+b^{2}} \xi\right)\right]=\hat{\mathbb{E}}\left[\varphi\left(\sqrt{a^{2}+b^{2}} K \xi\right)\right] .
$$

Hence $K \xi$ is also normally distributed. Relation (2.5) is easy to check.

By Daniell-Stone Theorem, we have the following robust representation theorem of sublinear expectations which is quite useful.

Theorem 11. (see Peng [15]) Let $(\Omega, \mathcal{H}, \hat{\mathbb{E}})$ be a sublinear expectation space satisfying the regular property. Then there exists a weakly compact set $\mathcal{P}$ of probability measures defined on $(\Omega, \sigma(\mathcal{H}))$ such that

$$
\hat{\mathbb{E}}[X]=\max _{P \in \mathcal{P}} E_{P}[X] \text { for all } X \in \mathcal{H},
$$

where $\sigma(\mathcal{H})$ is the $\sigma$-algebra generated by all functions in $\mathcal{H} . \mathcal{P}$ is called the the family of probability measures that represents $\hat{\mathbb{E}}$.

Let $\mathcal{P}$ be a weakly compact set that represents $\hat{\mathbb{E}}$. For this $\mathcal{P}$, we define capacity

$$
c(A):=\sup _{P \in \mathcal{P}} P(A), \quad A \in \mathcal{B}(\Omega) .
$$

A set $A \subset \mathcal{B}(\Omega)$ is called polar if $c(A)=0$. A property holds "quasi-surely" (q.s.) if it holds outside a polar set. In the following, we do not distinguish two random variables $X$ and $Y$ if $X=Y$ q.s. 
Definition 12. Let $\Gamma$ be a set of indices. A family of $\mathbb{R}^{d}$-valued random vectors $\left(X_{\gamma}\right)_{\gamma \in \Gamma}$ is called a $d$-dimensional stochastic process indexed by $\Gamma$, or defined on $\Gamma$, if for each $\gamma \in \Gamma, X_{\gamma} \in \mathcal{H}^{d}$. Let $\left(X_{\gamma}\right)_{\gamma \in \Gamma}$ and $\left(Y_{\gamma}\right)_{\gamma \in \Gamma}$ be two processes indexed by $\Gamma$. $Y$ is called a quasi-modification of $X$ if for all $\gamma \in \Gamma, X_{\gamma}=Y_{\gamma}$ q.s.

\section{G-GAUSSIAN RANDOM FIELDS}

\subsection{A general setting of random fields defined on a nonlinear expectation space.}

Definition 13. Let $(\Omega, \mathcal{H}, \hat{\mathbb{E}})$ be a nonlinear expectation space and $\Gamma$ be a parameter set. An $m$-dimensional random field on $(\Omega, \mathcal{H}, \hat{\mathbb{E}})$ is a family of random vectors $W=\left(W_{\gamma}\right)_{\gamma \in \Gamma}$ such that $W_{\gamma} \in \mathcal{H}^{m}$ for each $\gamma \in \Gamma$.

Let us denote the family of all sets of finite indices by

$$
\mathcal{J}_{\Gamma}:=\left\{\underline{\gamma}=\left(\gamma_{1}, \cdots, \gamma_{n}\right): \forall n \in \mathbb{N}, \gamma_{1}, \cdots, \gamma_{n} \in \Gamma, \gamma_{i} \neq \gamma_{j} \text { for } i \neq j, 1 \leq i, j \leq n\right\} .
$$

Now we give the notion of finite dimensional distribution of the random field $W$.

Definition 14. For each $\underline{\gamma}=\left(\gamma_{1}, \cdots, \gamma_{n}\right) \in \mathcal{J}_{\Gamma}$, let $\mathbb{F}_{\underline{\gamma}}$ be a nonlinear expectation on $\left(\mathbb{R}^{n \times m}, C_{\text {l.Lip }}\left(\mathbb{R}^{n \times m}\right)\right)$. We call $\left(\mathbb{F}_{\underline{\gamma}}\right)_{\underline{\gamma} \in \mathcal{J}_{\Gamma}}$ a system of finite dimensional distributions on $\mathbb{R}^{m}$ with respect to $\Gamma$.

Let $\left(W_{\gamma}\right)_{\gamma \in \Gamma}$ be an $m$-dimensional random field defined on a nonlinear expectation space $(\Omega, \mathcal{H}, \hat{\mathbb{E}})$. For each $\underline{\gamma}=\left(\gamma_{1}, \cdots, \gamma_{n}\right) \in \mathcal{J}_{\Gamma}$ and the corresponding ramdom variable $W_{\underline{\gamma}}=\left(W_{\gamma_{1}}, \cdots, W_{\gamma_{n}}\right)$, we define a functional on $C_{l . L i p}\left(\mathbb{R}^{n \times m}\right)$ by

$$
\mathbb{F}_{W_{\underline{\gamma}}}[\varphi]=\hat{\mathbb{E}}\left[\varphi\left(W_{\underline{\gamma}}\right)\right], \quad \varphi \in C_{l . L i p}\left(\mathbb{R}^{n \times m}\right) .
$$

Then the triple $\left(\mathbb{R}^{m \times n}, C_{\text {l.Lip }}\left(\mathbb{R}^{m \times n}\right), \mathbb{F}_{W_{\gamma}}\right)$ constitute a nonlinear expectation space. We call $\left(\mathbb{F}_{W_{\underline{\gamma}}}\right)_{\gamma \in \mathcal{J}_{\Gamma}}$ the family of finite dimensional distributions of $\left(W_{\gamma}\right)_{\gamma \in \Gamma}$.

Let $\left(\bar{W}_{\gamma}^{(\overline{1})}\right)_{\gamma \in \Gamma}$ and $\left(W_{\gamma}^{(2)}\right)_{\gamma \in \Gamma}$ be two $m$-dimensional random fields defined on nonlinear expectation spaces $\left(\Omega_{1}, \mathcal{H}_{1}, \hat{\mathbb{E}}_{1}\right)$ and $\left(\Omega_{2}, \mathcal{H}_{2}, \hat{\mathbb{E}}_{2}\right)$, respectively. They are said to be identically distributed, denoted by $\left(W_{\gamma}^{(1)}\right)_{\gamma \in \Gamma} \stackrel{d}{=}\left(W_{\gamma}^{(2)}\right)_{\gamma \in \Gamma}$, or simply $W^{(1)} \stackrel{d}{=} W^{(2)}$, if for each $\underline{\gamma}=\left(\gamma_{1}, \cdots, \gamma_{n}\right) \in \mathcal{J}_{\Gamma}, W_{\underline{\gamma}}^{(1)} \stackrel{d}{=} W_{\underline{\underline{\gamma}}}^{(2)}$, i.e.,

$$
\hat{\mathbb{E}}_{1}\left[\varphi\left(W_{\underline{\gamma}}^{(1)}\right)\right]=\hat{\mathbb{E}}_{2}\left[\varphi\left(W_{\underline{\gamma}}^{(2)}\right)\right] \text { for each } \varphi \in C_{l . L i p}\left(\mathbb{R}^{n \times m}\right) .
$$

For any given $m$-dimensional random field $W=\left(W_{\gamma}\right)_{\gamma \in \Gamma}$, the family of its finite dimensional distributions satisfies the following properties of consistency:

(1) Compatibility: For each $\left(\gamma_{1}, \cdots, \gamma_{n}, \gamma_{n+1}\right) \in \mathcal{J}_{\Gamma}$ and $\varphi \in C_{l . L i p}\left(\mathbb{R}^{n \times m}\right)$,

$$
\mathbb{F}_{W_{\gamma_{1}}, \cdots, W_{\gamma_{n}}}[\varphi]=\mathbb{F}_{W_{\gamma_{1}}, \cdots, W_{\gamma_{n}}, W_{\gamma_{n+1}}}[\widetilde{\varphi}],
$$

where the function $\widetilde{\varphi}$ is a function on $\mathbb{R}^{m \times(n+1)}$ defined by $\widetilde{\varphi}\left(y_{1}, \cdots, y_{n}, y_{n+1}\right)=$ $\varphi\left(y_{1}, \cdots, y_{n}\right)$, for any $y_{1}, \cdots, y_{n}, y_{n+1} \in \mathbb{R}^{m}$

(2) Symmetry: For each $\left(\gamma_{1}, \cdots, \gamma_{n}\right) \in \mathcal{J}_{\Gamma}, \varphi \in C_{l . L i p}\left(\mathbb{R}^{n \times m}\right)$ and each permutation $\pi$ of $\{1, \cdots, n\}$,

$$
\mathbb{F}_{W_{\gamma_{\pi(1)}}, \cdots, W_{\gamma_{\pi(n)}}}[\varphi]=\mathbb{F}_{W_{\gamma_{1}}, \cdots, W_{\gamma_{n}}}\left[\varphi_{\sigma}\right],
$$


where we denote $\varphi_{\pi}\left(y_{1}, \cdots, y_{n}\right)=\varphi\left(y_{\pi(1)}, \cdots, y_{\pi(n)}\right)$.

According to the family of finite dimensional distributions, we can generalize the classical Kolmogorov's existence theorem to the situation of a sublinear expectation space, which is a variant of Theorem 3.8 in Peng [17.

Theorem 15. Let $\left\{\mathbb{F}_{\gamma}, \gamma \in \mathcal{J}_{\Gamma}\right\}$ be a family of finite dimensional distributions on $\mathbb{R}^{m}$ satisfying the compatibitity condition (3.1) and the symmetry condition (3.2). Then there exists an $m$-dimensional random field $W=\left(W_{\gamma}\right)_{\gamma \in \Gamma}$ defined on a nonlinear expectation space $(\Omega, \mathcal{H}, \hat{\mathbb{E}})$ whose family of finite dimensional distributions coincides with $\left\{\mathbb{F}_{\underline{\gamma}}, \underline{\gamma} \in \mathcal{J}_{\Gamma}\right\}$. If we assume moreover that each $\mathbb{F}_{\underline{\gamma}}$ in $\left\{\mathbb{F}_{\underline{\gamma}}, \underline{\gamma} \in \mathcal{J}_{\Gamma}\right\}$ is sublinear, then the corresponding expectation $\hat{\mathbb{E}}$ on the space of random variables $(\Omega, \mathcal{H})$ is also sublinear.

Proof. Denote by $\Omega=\left(\mathbb{R}^{m}\right)^{\Gamma}$ the space of all functions $\omega=\left(\omega_{\gamma}\right)_{\gamma \in \Gamma}$ from $\Gamma$ to $\mathbb{R}^{m}$. For each $\omega \in \Omega$, we denote by $W=\left(W_{\gamma}(\omega)=\omega_{\gamma}\right)_{\gamma \in \Gamma}$ and call $W$ the canonical process defined on $\Omega$. The space of random variables $\mathcal{H}$ is defined by

$$
\mathcal{H}=L_{i p}(\Omega)=\left\{\varphi\left(W_{\gamma_{1}}, \cdots, W_{\gamma_{n}}\right), \forall n \in \mathbb{N}, \gamma_{1}, \cdots, \gamma_{n} \in \Gamma, \varphi \in C_{l . L i p}\left(\mathbb{R}^{n \times m}\right)\right\} .
$$

Then for each random variable $\xi \in \mathcal{H}$ of the form $\xi=\varphi\left(W_{\gamma_{1}}, \cdots, W_{\gamma_{n}}\right)$, we define the corresponding nonlinear expectation by

$$
\hat{\mathbb{E}}[\xi]=\mathbb{F}_{\gamma_{1}, \cdots, \gamma_{n}}[\varphi]
$$

Since $\mathbb{F}_{\underline{\gamma}}, \underline{\gamma} \in \mathcal{J}_{\Gamma}$ satisfies the consistency conditions (3.1) and (3.2), thus the functional $\hat{\mathbb{E}}: \mathcal{H} \mapsto \mathbb{R}$ is consistently defined.

Since, for each $\underline{\gamma} \in \mathcal{J}_{\Gamma}$, the distribution $\mathbb{F}_{\underline{\gamma}}$ is monotone and constant preserving, hence the expectation $\hat{\mathbb{E}}[\cdot]$ satisfies the same properties. Namely, $\hat{\mathbb{E}}[\cdot]$ is a nonlinear expectation defined on $(\Omega, \mathcal{H})$. Obviously, the family of finite dimensional distributions of $\left(W_{\gamma}\right)_{\gamma \in \Gamma}$ is $\left(\mathbb{F}_{\underline{\gamma}}\right)_{\underline{\gamma} \in \mathcal{J}_{\Gamma}}$. Moreover, if for each $\underline{\gamma}=\left(\gamma_{1}, \cdots, \gamma_{n}\right) \in \mathcal{J}_{\Gamma}, \mathbb{F}_{\underline{\gamma}}$ is a sublinear expectation defined on $\left(\mathbb{R}^{m \times n}, C_{\text {l.Lip }}\left(\mathbb{R}^{m \times n}\right)\right)$, then $\hat{\mathbb{E}}$ is a sublinear expectation. The proof of the sub-additivity of $\hat{\mathbb{E}}[\cdot]$ is as follows: let $\xi=\varphi\left(W_{\gamma_{1}}, \cdots, W_{\gamma_{i}}\right)$ and $\eta=\psi\left(W_{\iota_{1}}, \cdots, W_{\iota_{j}}\right)$ be two random variables in $\mathcal{H}$, with $\varphi \in C_{l . L i p}\left(\mathbb{R}^{m \times i}\right)$ and $\psi \in C_{l . L i p}\left(\mathbb{R}^{m \times j}\right)$. Without loss of generality, assume that $\gamma_{h} \neq \iota_{k}$, for $h=1, \cdots, i, k=1, \cdots, j$. Then, from the definition of $\hat{\mathbb{E}}$,

$$
\begin{aligned}
\hat{\mathbb{E}}[\xi+\eta] & =\mathbb{F}_{\gamma_{1}, \cdots, \gamma_{i}, \iota_{1}, \cdots, \iota_{j}}[\widetilde{\varphi}+\widetilde{\psi}] \leq \mathbb{F}_{\gamma_{1}, \cdots, \gamma_{i}, \iota_{1}, \cdots, \iota_{j}}[\widetilde{\varphi}]+\mathbb{F}_{\gamma_{1}, \cdots, \gamma_{i}, \iota_{1}, \cdots, \iota_{j}}[\widetilde{\psi}] \\
& =\mathbb{F}_{\gamma_{1}, \cdots, \gamma_{i}}[\varphi]+\mathbb{F}_{\iota_{1}, \cdots, \iota_{j}}[\psi]=\hat{\mathbb{E}}[\xi]+\hat{\mathbb{E}}[\eta]
\end{aligned}
$$

where we set

$$
\begin{aligned}
& \widetilde{\varphi}\left(\gamma_{1}, \cdots, \gamma_{i}, \iota_{1}, \cdots, \iota_{j}\right)=\varphi\left(\gamma_{1}, \cdots, \gamma_{i}\right), \\
& \widetilde{\psi}\left(\gamma_{1}, \cdots, \gamma_{i}, \iota_{1}, \cdots, \iota_{j}\right)=\psi\left(\iota_{1}, \cdots, \iota_{j}\right) .
\end{aligned}
$$

The positive homogeneity of $\hat{\mathbb{E}}$ is directly obtained from the property of $\mathbb{F}_{\underline{\gamma}}, \underline{\gamma} \in \mathcal{J}_{\Gamma}$. The proof is complete. 


\subsection{Gaussian random fields under a sublinear expectation space.}

Definition 16. Let $\left(W_{\gamma}\right)_{\gamma \in \Gamma}$ be an $m$-dimensional random field on a sublinear expectation space $(\Omega, \mathcal{H}, \hat{\mathbb{E}})$. $\left(W_{\gamma}\right)_{\gamma \in \Gamma}$ is called an $m$-dimensional $G$-Gaussian random field if for each $\underline{\gamma}=\left(\gamma_{1}, \cdots, \gamma_{n}\right) \in \mathcal{J}_{\Gamma}$, the following $n \times m$-dimensional random vector

$$
\begin{gathered}
W_{\underline{\gamma}}=\left(W_{\gamma_{1}}, \cdots, W_{\gamma_{n}}\right)=\left(W_{\gamma_{1}}^{(1)}, \cdots W_{\gamma_{1}}^{(m)}, \cdots, W_{\gamma_{n}}^{(1)}, \cdots, W_{\gamma_{n}}^{(m)}\right), W_{\gamma_{i}}^{(j)} \in \mathcal{H}, \\
i=1, \cdots, n, \quad j=1, \cdots, m,
\end{gathered}
$$

is $G$-normally distributed. Namely,

$$
a W_{\underline{\gamma}}+b \bar{W}_{\underline{\gamma}} \stackrel{d}{=} \sqrt{a^{2}+b^{2}} W_{\underline{\gamma}}, \quad \text { for any } a, b \geq 0,
$$

where $\bar{W}_{\underline{\gamma}}$ is an independent copy of $W_{\underline{\gamma}}$.

For each $\underline{\gamma}=\left(\gamma_{1}, \cdots, \gamma_{n}\right) \in \mathcal{J}_{\Gamma}$, we define

$$
G_{W_{\underline{\gamma}}}(Q)=\frac{1}{2} \hat{\mathbb{E}}\left[\left\langle Q W_{\underline{\gamma}}, W_{\underline{\gamma}}\right\rangle\right], \quad Q \in \mathbb{S}(n \times m),
$$

where $\mathbb{S}(n \times m)$ denotes the collection of all $(n \times m) \times(n \times m)$ symmetric matrices. Then $\left(G_{W_{\underline{\gamma}}}\right)_{\underline{\gamma} \in \mathcal{J}_{\Gamma}}$ constitutes a family of functions:

$$
G_{W_{\underline{\gamma}}}: \mathbb{S}(n \times m) \mapsto \mathbb{R}, \quad \underline{\gamma}=\left(\gamma_{1}, \cdots, \gamma_{n}\right), \gamma_{i} \in \Gamma, n \in \mathbb{N},
$$

which satisfies the sublinear and monotone properties in the sense of (2.2).

According to Proposition 8 , the corresponding distribution $\mathbb{F}_{W_{\gamma}}$ of this $G$-normally distributed random vector $W_{\underline{\gamma}}$ is uniquely determined by the function $G_{W_{\underline{\gamma}}}$. Clearly, $\left\{G_{W_{\underline{\gamma}}}\right\}_{\underline{\gamma} \in \mathcal{J}_{\Gamma}}$ is a family of moñotone sublinear and continuous functions satisfying the properties of consistency in the following sense:

(1) Compatibility: For any $\left(\gamma_{1}, \cdots, \gamma_{n}, \gamma_{n+1}\right) \in \mathcal{J}_{\Gamma}$ and $Q=\left(q_{i j}\right)_{i, j=1}^{n \times m} \in \mathbb{S}(n \times$ $m$ )

$$
G_{W \gamma_{1}, \cdots, W_{\gamma_{n}}, W_{\gamma_{n+1}}}(\bar{Q})=G_{W \gamma_{1}, \cdots, W_{\gamma_{n}}}(Q),
$$

where $\bar{Q}=\left(\begin{array}{cc}Q & 0 \\ 0 & 0\end{array}\right) \in \mathbb{S}((n+1) \times m)$;

(2) Symmetry: For any permutation $\pi$ of $\{1, \cdots, n\}$,

$$
G_{W_{\gamma_{\pi(1)}}, \cdots, W_{\gamma_{\pi(n)}}}(Q)=G_{W_{\gamma_{1}}, \cdots, W_{\gamma_{n}}}\left(\pi^{-1}(Q)\right)
$$

where the mapping $\pi^{-1}: \mathbb{S}(n \times m) \mapsto \mathbb{S}(n \times m)$ is defined by

$$
\left(\pi^{-1}(Q)\right)_{i j}=\left(q_{\pi^{-1}(i) \pi^{-1}(j)}\right), i, j=1, \cdots,(n \times m), \quad Q=\left(q_{i j}\right)_{i, j=1}^{n \times m} \in \mathbb{S}(n \times m) .
$$

A very inttersting inverse problem is how to construct a $G$-Gaussian field in a sublinear expectation space if a above type of family of sublinear functions $\left(G_{\underline{\gamma}}\right)_{\underline{\gamma} \in \mathcal{J}_{\Gamma}}$ is given.

Theorem 17. Let $\left(G_{\underline{\gamma}}\right)_{\underline{\gamma} \in \mathcal{J}_{\Gamma}}$ be a family of real valued functions such that, for each $\underline{\gamma}=\left(\gamma_{1}, \cdots, \gamma_{n}\right) \in \mathcal{J}_{\Gamma}$, the real function $G_{\underline{\gamma}}$ is defined on $\mathbb{S}(n \times m) \mapsto \mathbb{R}$ and satisfies the same monotone and sublinear property of type (2.2). Moreover, this family $\left(G_{\underline{\gamma}}\right)_{\underline{\gamma} \in \mathcal{J}_{\Gamma}}$ satisfies the same compatibility condition (3.3) and symmetry condition (3.4). Then 
there exists an $m$-dimensional $G$-Gaussian random field $\left(W_{\gamma}\right)_{\gamma \in \Gamma}$ on a sublinear expectation space $(\Omega, \mathcal{H}, \hat{\mathbb{E}})$ such that for each $\underline{\gamma}=\left(\gamma_{1}, \cdots, \gamma_{n}\right) \in \mathcal{J}_{\Gamma}, W_{\underline{\gamma}}=\left(W_{\gamma_{1}}, \cdots, W_{\gamma_{n}}\right)$ is $G$-normally distributed, i.e.

$$
G_{W_{\underline{\gamma}}}(Q)=\frac{1}{2} \hat{\mathbb{E}}\left[\left\langle Q W_{\underline{\gamma}}, W_{\underline{\gamma}}\right\rangle\right]=G_{\underline{\gamma}}(Q), \quad \text { for any } Q \in \mathbb{S}(n \times m) .
$$

Furthermore, if there exists another Gaussian random field $\left(\bar{W}_{\gamma}\right)_{\gamma \in \Gamma}$, with the same index set $\Gamma$, defined on a sublinear expectation space $(\bar{\Omega}, \overline{\mathcal{H}}, \overline{\mathbb{E}})$ such that for each $\underline{\gamma} \in \mathcal{J}_{\Gamma}$, $\bar{W}_{\underline{\gamma}}$ is G-normally distributed with the same generating function, namely,

$$
\frac{1}{2} \overline{\mathbb{E}}\left[\left\langle Q \bar{W}_{\underline{\gamma}}, \bar{W}_{\underline{\gamma}}\right\rangle\right]=G_{\underline{\gamma}}(Q) \quad \text { for any } Q \in \mathbb{S}(n \times m) .
$$

Then we have $W \stackrel{d}{=} \bar{W}$.

Proof. For each $\underline{\gamma}=\left(\gamma_{1}, \cdots, \gamma_{n}\right) \in \mathcal{J}_{\Gamma}$, we denote by $\mathbb{F}_{W_{\underline{\gamma}}}$ the sublinear distribution uniquely determined by $G_{\underline{\gamma}}$. Since the family of monotone sublinear functions $G_{W_{\underline{\gamma}}}$ satisfies the conditions of the consistency (3.3), (3.4). It follows that the family of $G^{-}$ normal distributions $\mathbb{F}_{W_{\underline{\gamma}}}[\cdot]$ satisfies the conditions of consistency (3.1), (3.2). Thus we can follow the construction procedure in the proof of Theorem 15 to construct a random field $W=\left(W_{\gamma}\right)_{\gamma \in \Gamma}$ on a complete sublinear expectation space $(\Omega, \mathcal{H}, \hat{\mathbb{E}})$, under which the family of finite dimensional distributions coincides with $\left\{\mathbb{F}_{W_{\underline{\gamma}}}\right\}_{\underline{\underline{\gamma}} \in \mathcal{J}_{\Gamma}}$. Obviously, $W$ is a Gaussian random field under $\hat{\mathbb{E}}$.

Assume that there exists another $G$-Gaussian random field $\bar{W}$ with the same family of generating functions. Then $W$ and $\bar{W}$ have the same finite-dimensional distributions, which implies $W \stackrel{d}{=} \bar{W}$. The proof is complete.

For each $p \geq 1$, let $\mathbb{L}_{G}^{p}(W)$ be the completion of $\mathcal{H}$ under the Banach norm $\|\cdot\|_{\mathbb{L}_{G}^{p}}:=$ $\left(\hat{\mathbb{E}}\left[|\cdot|^{p}\right]\right)^{1 / p}$. Then the sublinear expectation $\hat{\mathbb{E}}$ can be extended continuously to $\mathbb{L}_{G}^{p}(W)$ and $\left(\Omega, \mathbb{L}_{G}^{p}(W), \hat{\mathbb{E}}\right)$ forms a complete sublinear expectation space.

Remark 18. If $\Gamma=\mathbb{R}^{+}, W=\left(W_{\gamma}\right)_{\gamma \in \Gamma}$ becomes a $G$-Gaussian process which has been studied in Peng [17].

\section{Spatial White noise Under Sublinear Expectations}

In this section, we formulate a spatial white noise and then develop the corresponding stochastic integral.

Definition 19. Let $(\Omega, \mathcal{H}, \hat{\mathbb{E}})$ be a sublinear expectation space and $\Gamma=\mathcal{B}_{0}\left(\mathbb{R}^{d}\right)=$ $\left\{A \in \mathcal{B}\left(\mathbb{R}^{d}\right), \lambda_{A}<\infty\right\}$, where $\lambda_{A}$ denotes the Lebesgue measure of $A \in \mathcal{B}\left(\mathbb{R}^{d}\right)$. A 1-dimensional G-Gaussian random field $\mathbb{W}=\left\{\mathbb{W}_{A}, A \in \Gamma\right\}$ is called a 1-dimensional $G$-white noise if

(i) $\hat{\mathbb{E}}\left[\mathbb{W}_{A}^{2}\right]=\bar{\sigma}^{2} \lambda_{A},-\hat{\mathbb{E}}\left[-\mathbb{W}_{A}^{2}\right]=\underline{\sigma}^{2} \lambda_{A}$, for all $A \in \Gamma$; 
(ii) For each $A_{1}, A_{2} \in \Gamma, A_{1} \cap A_{2}=\emptyset$, we have

$$
\begin{aligned}
& \hat{\mathbb{E}}\left[\mathbb{W}_{A_{1}} \mathbb{W}_{A_{2}}\right]=\hat{\mathbb{E}}\left[-\mathbb{W}_{A_{1}} \mathbb{W}_{A_{2}}\right]=0, \\
& \hat{\mathbb{E}}\left[\left(\mathbb{W}_{A_{1} \cup A_{2}}-\mathbb{W}_{A_{1}}-\mathbb{W}_{A_{2}}\right)^{2}\right]=0,
\end{aligned}
$$

where $0 \leq \underline{\sigma}^{2} \leq \bar{\sigma}^{2}$ are any given numbers.

Remark 20. The definition implies that the distribution of a $G$-white noise has only two parameters $\bar{\sigma}^{2}$ and $\underline{\sigma}^{2}$. In fact we can also set $\bar{\sigma}^{2}=1, \underline{\sigma}^{2} \leq 1$ to make it as a 1-parameter field. On the other hand, for any given pair of numbers $0 \leq \underline{\sigma}^{2} \leq \bar{\sigma}^{2}$, one can construct a 1-dimensional random field $\mathbb{W}=\left\{\mathbb{W}_{A}, A \in \Gamma\right\}$ with $\Gamma=\mathcal{B}_{0}\left(\mathbb{R}^{d}\right)$ defined on a sublinear expectation space $(\Omega, \mathcal{H}, \hat{\mathbb{E}})$ satisfying conditions (i)-(ii).

We denote by $\mathbb{L}_{G}^{2}(\mathbb{W})$ the completion of $\mathcal{H}$ under the Banach norm $\|\cdot\|_{\mathbb{L}_{G}^{2}}:=$ $\left(\hat{\mathbb{E}}\left[|\cdot|^{2}\right]\right)^{1 / 2}$. Then the sublinear expectation $\hat{\mathbb{E}}$ can be extended continuously to $\mathbb{L}_{G}^{2}(\mathbb{W})$ and $\left(\Omega, \mathbb{L}_{G}^{2}(\mathbb{W}), \hat{\mathbb{E}}\right)$ forms a complete sublinear expectation space.

4.1. Existence of $G$-white noise. In the following, we turn to construct a spatial $G$-white noise on some sublinear expectation space. According to Theorem 17, it is sufficient to define an appropriate family of sublinear and monotone functions $\left(G_{\gamma}\right)_{\gamma \in \mathcal{J}_{\Gamma}}$ such that the $G$-Gaussian random field generated by $\left(G_{\underline{\gamma}}\right)_{\underline{\gamma} \in \mathcal{J}_{\Gamma}}$ satisfies conditions (i)-(ii) in Definition 19 for $G$-white noise.

For each $\underline{\gamma}=\left(A_{1}, \cdots, A_{n}\right), A_{j} \in \Gamma=\mathcal{B}_{0}\left(\mathbb{R}^{d}\right)$, we define a mapping $G_{\underline{\gamma}}(\cdot): \mathbb{S}(n) \mapsto \mathbb{R}$ as follows:

$$
G_{A_{1}, \cdots, A_{n}}(Q)=G\left(\sum_{i, j=1}^{n} q_{i j} \lambda_{A_{i} \cap A_{j}}\right), Q=\left(q_{i j}\right)_{i, j=1}^{n} \in \mathbb{S}(n) .
$$

where $G(a)=\frac{1}{2} \bar{\sigma}^{2} a^{+}-\frac{1}{2} \underline{\sigma}^{2} a^{-}$for $a \in \mathbb{R}$. Here $\bar{\sigma}^{2} \geq \underline{\sigma}^{2}$ are two given nonnegative parameters.

Proposition 21. For each $\underline{\gamma}=\left(A_{1}, \cdots, A_{n}\right), A_{j} \in \Gamma=\mathcal{B}_{0}\left(\mathbb{R}^{d}\right)$, the function $G_{\gamma}(\cdot)$ defined as (4.3) is a sublinear and monotone real function defined on $\mathbb{S}(n)$, namely it satisfies the condition (2.2). Moreover, the family $\left\{G_{\underline{\gamma}}\right\}_{\underline{\underline{\gamma}} \in \mathcal{J}_{\Gamma}}$ satisfies the conditions of consistency given in (3.3) and (3.4).

Proof. The property that $G_{\underline{\gamma}}$ satisfies relation (2.2) follows from the monotone and sublinear properties of the function $G=G(a), a \in \mathbb{R}$, and the property that for each $A_{1}, \cdots, A_{n} \in \mathcal{B}_{0}\left(\mathbb{R}^{d}\right)$,

$$
\sum_{i, j=1}^{n} q_{i j} \lambda_{A_{i} \cap A_{j}} \geq 0, \quad \text { if } Q \geq 0 .
$$

The compatibility of the type (3.3) is checked as follows: let

$$
\bar{Q}=\left(\begin{array}{cc}
Q & 0 \\
0 & 0
\end{array}\right) \in \mathbb{S}(n+1)
$$


and let $A_{1}, \cdots, A_{n}, A_{n+1} \in \mathcal{B}_{0}\left(\mathbb{R}^{d}\right)$. Noting that $q_{i(n+1)}=q_{(n+1) i}=0, i=1, \cdots, n+1$, by (4.3), we have

$$
G_{A_{1}, \cdots, A_{n+1}}(\bar{Q})=G_{A_{1}, \cdots, A_{n}}(Q)
$$

which is (3.3). Let $\pi(\cdot)$ be a permutation of $\{1,2, \cdots, n\}$. By (4.3), we can check that

$$
G_{A_{1}, \cdots, A_{n}}(Q)=G_{A_{\pi(1)}, \cdots, A_{\pi(1)}}(\pi(Q)),
$$

where $\pi(Q)=\left(q_{\pi(i) \pi(j)}\right)_{i, j=1}^{n}$. Thus (3.4) holds. The proof is complete.

Now we present the existence of white noise under the sublinear expectation.

Theorem 22. For each given sublinear and monotone function

$$
G(a)=\frac{1}{2}\left(\bar{\sigma}^{2} a^{+}-\underline{\sigma}^{2} a^{-}\right), a \in \mathbb{R},
$$

let the family of generating functions $\left\{G_{\underline{\gamma}}(\cdot): \underline{\gamma}=\left(A_{1}, \cdots, A_{n}\right) \in \mathcal{J}_{\Gamma}\right\}$ be defined as in (4.3). Then there exists a 1-dimensional $G$-Gaussian random field $\left(\mathbb{W}_{\gamma}\right)_{\gamma \in \Gamma}$ on a complete sublinear expectation space $\left(\Omega, \mathbb{L}_{G}^{2}(\mathbb{W}), \hat{\mathbb{E}}\right)$ such that, for each $\gamma=\left(A_{1}, \cdots, A_{n}\right) \in$ $\mathcal{J}_{\Gamma}, W_{\underline{\gamma}}=\left(\mathbb{W}_{A_{1}}, \cdots, \mathbb{W}_{A_{n}}\right)$ is G-normally distributed, i.e.,

$$
G_{\mathbb{W}_{\underline{\gamma}}}(Q)=\frac{1}{2} \hat{\mathbb{E}}\left[\left\langle Q \mathbb{W}_{\underline{\underline{\gamma}}}, \mathbb{W}_{\underline{\underline{\gamma}}}\right\rangle\right]=G\left(\sum_{i, j=1}^{n} q_{i j} \lambda_{A_{i} \cap A_{j}}\right), \quad \text { for any } Q=\left(q_{i j}\right)_{i, j=1}^{n} \in \mathbb{S}(n) .
$$

Moreover, $\left(\mathbb{W}_{\gamma}\right)_{\gamma \in \Gamma}$ is also a spatial $G$-white noise under $\left(\Omega, \mathbb{L}_{G}^{2}(\mathbb{W}), \hat{\mathbb{E}}\right)$, namely, conditions (i) and (ii) of Definition 19 are satisfied. If $\left(\mathbb{W}_{\gamma}\right)_{\gamma \in \Gamma}$ is another G-white noise with the same sublinear function $G$, then its family of generating function $\left\{G_{\overline{\mathbb{W}}_{\underline{\gamma}}}\right\}_{\gamma \in \mathcal{J}_{\Gamma}}$ coincides with the one defined in 4.3.).

Proof. Since the compatibility and symmetry conditions of $\left\{G_{\underline{\gamma}}\right\}_{\underline{\gamma} \in \mathcal{J}_{\Gamma}}$ has already been proved, it then follows from Lemma 21 and Theorem 17 that the existence and uniqueness of the $G$-Gaussian random field $\mathbb{W}$ in a complete sublinear expectation space $\left(\Omega, \mathbb{L}_{G}^{2}(\mathbb{W}), \hat{\mathbb{E}}\right)$ with the family of generating functions defined by (4.3) hold. We only need to prove that $\mathbb{W}$ is a $G$-white noise. It suffices to verify that the $G$-random field $\mathbb{W}$ induced by the family of generating functions defined in (4.3) satisfies conditions (i)-(ii) of Definition 19,

For any $A \in \Gamma$,

$$
\hat{\mathbb{E}}\left[\mathbb{W}_{A}^{2}\right]=2 G_{A}(1)=2 G\left(\lambda_{A}\right)=\bar{\sigma}^{2} \lambda_{A}
$$

Similarly, we have $\hat{\mathbb{E}}\left[-\mathbb{W}_{A}^{2}\right]=-\underline{\sigma}^{2} \lambda_{A}$, which verifies (i). Now consider relation (4.1) in (ii). Let $A_{1}, A_{2} \in \Gamma$ be such that $A_{1} \cap A_{2}=\emptyset$. On the other hand, we can choose a $2 \times 2$ symmetric matrix $Q=\left(q_{i j}\right)_{i, j=1}^{2}$ to express

$$
\hat{\mathbb{E}}\left[\mathbb{W}_{A_{1}} \mathbb{W}_{A_{2}}\right]=\hat{\mathbb{E}}\left[\sum_{i, j=1}^{2} q_{i j} \mathbb{W}_{A_{i}} \mathbb{W}_{A_{j}}\right]
$$

where we set

$$
Q=\left(q_{i j}\right)_{i, j=1}^{2}=\left[\begin{array}{cc}
0 & \frac{1}{2} \\
\frac{1}{2} & 0
\end{array}\right] .
$$


It then follows from (4.3) that

$$
\hat{\mathbb{E}}\left[\mathbb{W}_{A_{1}} \mathbb{W}_{A_{2}}\right]=\hat{\mathbb{E}}\left[\sum_{i, j=1}^{2} q_{i j} \mathbb{W}_{A_{i}} \mathbb{W}_{A_{j}}\right]=G\left(\sum_{i, j=1}^{2} q_{i j} \lambda_{A_{i} \cap A_{j}}\right)=0
$$

Similarly, we can get $\hat{\mathbb{E}}\left[-\mathbb{W}_{A_{1}} \mathbb{W}_{A_{2}}\right]=0$, thus (4.1) holds.

To prove (4.2), let $A_{1}, A_{2} \in \Gamma$ be such that $A_{1} \cap A_{2}=\emptyset$ and denote $A_{3}=A_{1} \cup A_{2}$. Then

$$
\hat{\mathbb{E}}\left[\left(\mathbb{W}_{A_{1}}+\mathbb{W}_{A_{2}}-\mathbb{W}_{A_{3}}\right)^{2}\right]=\hat{\mathbb{E}}\left[\sum_{i, j=1}^{3} q_{i j} \mathbb{W}_{A_{i}} \mathbb{W}_{A_{j}}\right]
$$

where we denote

$$
Q=\left(q_{i j}\right)_{i, j=1}^{3}=\left[\begin{array}{ccc}
1 & 1 & -1 \\
1 & 1 & -1 \\
-1 & -1 & 1
\end{array}\right]
$$

We then apply (4.3) to get

$$
\hat{\mathbb{E}}\left[\sum_{i, j=1}^{3} q_{i j} \mathbb{W}_{A_{i}} \mathbb{W}_{A_{j}}\right]=G\left(\sum_{i, j=1}^{3} q_{i j} \lambda_{A_{i} \cup A_{j}}\right)=G\left(\lambda_{A_{1}}+\lambda_{A_{2}}+\lambda_{A_{1} \cap A_{2}}-2\left(\lambda_{A_{2}}+\lambda_{A_{1}}\right)\right]=0
$$

which yields (4.2).

Now let $\left\{\mathbb{W}_{\gamma}\right\}_{\gamma \in \Gamma}$ be a $G$-white noise, associated with the same function $G$, on a complete sublinear expectation space $\left(\bar{\Omega}, \mathbb{L}_{G}^{2}(\overline{\mathbb{W}}), \overline{\mathbb{E}}\right)$. Then for each $\underline{\gamma}=\left(A_{1}, \cdots, A_{n}\right) \in$ $\mathcal{J}_{\Gamma}, Q=\left(q_{i j}\right)_{i, j=1}^{n} \in \mathbb{S}(n)$,

$$
G_{\overline{\mathbb{W}}_{\underline{\underline{\gamma}}}}(Q)=\frac{1}{2} \hat{\mathbb{E}}\left[\left\langle Q \overline{\mathbb{W}}_{\underline{\gamma}}, \overline{\mathbb{W}}_{\underline{\underline{\gamma}}}\right\rangle\right]=G\left(\sum_{i, j=1}^{n} q_{i j} \lambda_{A_{i} \cap A_{j}}\right)=G_{\underline{\gamma}}(Q) .
$$

Thus $\left\{\overline{\mathbb{W}}_{\gamma}\right\}_{\gamma \in \Gamma} \stackrel{d}{=}\left\{\mathbb{W}_{\gamma}\right\}_{\gamma \in \Gamma}$ and the proof is complete.

We can use a similar approach to prove that, for each $A, B \in \mathcal{B}_{0}\left(\mathbb{R}^{d}\right)$, we have

$$
\left.\hat{\mathbb{E}}\left(\mathbb{W}_{A \cup B}+\mathbb{W}_{A \cap B}-\mathbb{W}_{A}-\mathbb{W}_{B}\right)^{2}\right]=0 .
$$

Remark 23. From the above lemma, it follows directly that $\mathbb{W}_{A_{1} \cup A_{2}}=\mathbb{W}_{A_{1}}+\mathbb{W}_{A_{2}}$ $\mathbb{W}_{A_{1} \cap A_{2}}$ for any $A_{1}, A_{2} \in \Gamma$. Futhermore, if $\left\{A_{i}\right\}_{i=1}^{\infty}$ is a mutually disjoint sequence of $\Gamma$ such that $\sum_{i=1}^{\infty} \lambda_{A_{i}}<\infty$, then

$$
\mathbb{W}\left(\bigcup_{i=1}^{\infty} A_{i}\right)=\sum_{i=1}^{\infty} \mathbb{W}_{A_{i}} \in \mathbb{L}_{G}^{2}(\mathbb{W})
$$

A very important property of spatial $G$-white noise is that it is invariant under rotations and translations: 
Proposition 24. For each $p \in \mathbb{R}^{d}$ and $O \in \mathbb{O}(d):=\left\{O \in \mathbb{R}^{d \times d}, O^{T}=O^{-1}\right\}$, we set

$$
T_{p, O}(A)=\{O x+p: x \in A\} .
$$

Then, for each $A_{1}, \cdots, A_{n} \in \mathcal{B}_{0}\left(\mathbb{R}^{d}\right)$, we have

$$
\left(\mathbb{W}_{A_{1}}, \cdots, \mathbb{W}_{A_{n}}\right) \stackrel{d}{=}\left(\mathbb{W}_{T_{p, O}\left(A_{1}\right)}, \cdots, \mathbb{W}_{T_{p, O}\left(A_{n}\right)}\right) .
$$

Namely the finite dimensional distributions of $\mathbb{W}$ are invariant under rotations and translations.

Proof. It is a direct consequence of the well-know invariance of the Lebesgue measure under rotations and translations, i.e.,

$$
\lambda_{A}=\lambda_{T_{p, O}(A)}, \quad \forall(p, O) \in \mathbb{R}^{d} \times O(d), \quad A \in \mathcal{B}_{0}\left(\mathbb{R}^{d}\right) .
$$

According to Theorem 22, we can calculate and characterize each finite dimensional distribution of the $G$-white noise $\mathbb{W}$ by the nonlinear heat equations.

Let $\mathbb{W}$ be a $G$-white noise on a sublinear expectation space $(\Omega, \mathcal{H}, \hat{\mathbb{E}})$ with index $\Gamma=\mathcal{B}_{0}\left(\mathbb{R}^{d}\right)$. For each $\underline{\gamma}=\left(A_{1}, \cdots, A_{n}\right) \in \mathcal{J}_{\Gamma}, \mathbb{W}_{\underline{\gamma}}=\left(\mathbb{W}_{A_{1}}, \cdots, \mathbb{W}_{A_{n}}\right), n \in \mathbb{N}$, we have

$$
G_{\mathbb{W}_{\underline{\gamma}}}(Q)=\frac{1}{2} \hat{\mathbb{E}}\left[\left\langle Q \mathbb{W}_{\underline{\gamma}}, \mathbb{W}_{\underline{\gamma}}\right\rangle\right]=G_{\underline{\gamma}}(Q),
$$

where $Q=\left(q_{i j}\right) \in \mathbb{S}(n)$ and $G(q)=\frac{\bar{\sigma}^{2}}{2} q^{+}-\frac{\sigma^{2}}{2} q^{-}, q \in \mathbb{R}$. For any given $\varphi \in C_{l . L i p}\left(\mathbb{R}^{n}\right)$, set

$$
u\left(t, x_{1}, \cdots, x_{n}\right)=\hat{\mathbb{E}}\left[\varphi\left(x_{1}+\sqrt{t} \mathbb{W}_{A_{1}}, \cdots, x_{n}+\sqrt{t} \mathbb{W}_{A_{n}}\right)\right], \quad(t, x) \in[0, \infty) \times \mathbb{R} .
$$

Then by Proposition 9, $u$ is the unique viscosity solution of the Cauchy problem of the nonlinear heat equation:

$$
\partial_{t} u-G_{\underline{\gamma}}\left(D_{x x}^{2} u\right)=0,\left.\quad u\right|_{t=0}=\varphi\left(x_{1}, \cdots, x_{n}\right) .
$$

We then can obtain the nonlinear expected value by

$$
\hat{\mathbb{E}}\left[\varphi\left(\mathbb{W}_{A_{1}}, \cdots, \mathbb{W}_{A_{n}}\right)\right]=\left.u\right|_{t=1, x_{1}, \cdots, x_{n}=0} .
$$

4.2. Stochastic integrals with respect to a spatial white noise. Now let us define the stochastic integral with respect to $G$-white noise in three steps.

Let $\left\{\mathbb{W}_{\gamma}\right\}_{\gamma \in \Gamma}, \Gamma=\mathcal{B}_{0}\left(\mathbb{R}^{d}\right)$, be a 1-dimensional $G$-white noise with parameters $\underline{\sigma}^{2}, \bar{\sigma}^{2}$ on a complete sublinear expectation space $\left(\Omega, \mathbb{L}_{G}^{2}(\mathbb{W}), \hat{\mathbb{E}}\right)$.

Step 1: For each $A \in \Gamma, \lambda_{A}<\infty$, suppose $f: \mathbb{R}^{d} \rightarrow \mathbb{R}$ is an elementary function with the form $f(x)=\mathbf{1}_{A}(x), x \in \mathbb{R}^{d}$. We can define

$$
\int_{\mathbb{R}^{d}} f(x) \mathbb{W}(d x)=\int_{\mathbb{R}^{d}} \mathbf{1}_{A}(x) \mathbb{W}(d x)=\mathbb{W}_{A} .
$$

Step 2: For any simple function $f(x)=\sum_{i=1}^{n} a_{i} \mathbf{1}_{A_{i}}(x), n \in \mathbb{N}, a_{1}, \cdots, a_{n} \in \mathbb{R}, A_{1}, \cdots, A_{n} \in$ $\Gamma$, we define

$$
\int_{\mathbb{R}^{d}}\left(\sum_{i=1}^{n} a_{i} \mathbf{1}_{A_{i}}(x)\right) \mathbb{W}(d x)=\sum_{i=1}^{n} a_{i} \int_{\mathbb{R}^{d}} \mathbf{1}_{A_{i}}(x) \mathbb{W}(d x)=\sum_{i=1}^{n} a_{i} \mathbb{W}_{A_{i}}
$$


Step 3: Let $L^{2}\left(\mathbb{R}^{d}\right)$ denote the space of all functions $f: \mathbb{R}^{d} \rightarrow \mathbb{R}$ such that $\|f\|_{L^{2}}^{2}=$ $\int_{\mathbb{R}^{d}}|f(x)|^{2} d x<\infty$. By the following lemma, we can extend the stochastic integral to $L^{2}\left(\mathbb{R}^{d}\right)$.

Lemma 25. If $f: \mathbb{R}^{d} \rightarrow \mathbb{R}$ is a simple function, then

$$
\hat{\mathbb{E}}\left[\left|\int_{\mathbb{R}^{d}} f(x) \mathbb{W}(d x)\right|^{2}\right]=\bar{\sigma}^{2}\|f\|_{L^{2}}^{2}
$$

Proof. Without loss of generality, we suppose $f(x)=\sum_{i=1}^{n} a_{i} \mathbf{1}_{A_{i}}(x)$, where $a_{1}, \cdots, a_{n} \in$ $\mathbb{R}, A_{1}, \cdots, A_{n} \in \Gamma$ and $A_{i} \cap A_{j}=\emptyset$ if $i \neq j$. Then

$$
\begin{aligned}
\hat{\mathbb{E}}\left[\left|\int_{\mathbb{R}^{d}} f(x) \mathbb{W}(d x)\right|^{2}\right] & =\hat{\mathbb{E}}\left[\left|\sum_{i=1}^{n} a_{i} \mathbb{W}_{A_{i}}\right|^{2}\right]=\hat{\mathbb{E}}\left[\sum_{i=1}^{n} a_{i}^{2} \mathbb{W}_{A_{i}}^{2}\right] \\
& \leq \sum_{i=1}^{n} a_{i}^{2} \hat{\mathbb{E}}\left[\mathbb{W}_{A_{i}}^{2}\right]=\bar{\sigma}^{2} \sum_{i=1}^{n} a_{i}^{2} \lambda_{A_{i}}=\bar{\sigma}^{2}\|f\|_{L^{2}}^{2} .
\end{aligned}
$$

This lemma implies that the linear mapping

$$
\int_{\mathbb{R}^{d}} f(x) \mathbb{W}(d x): L^{2}\left(\mathbb{R}^{d}\right) \mapsto \mathbb{L}_{G}^{2}(\mathbb{W})
$$

is contract. We then can continuously extend this mapping to the the whole domain of $L^{2}\left(\mathbb{R}^{d}\right)$. We still denote this mapping by $\int_{\mathbb{R}^{d}} f(x) \mathbb{W}(d x)$ and call it integral of $f \in L^{2}\left(\mathbb{R}^{d}\right)$ with respect to the spatial white noise $\mathbb{W}$.

For each $B \subset \mathbb{R}^{d}, f \in L^{2}\left(\mathbb{R}^{d}\right)$, we can also define

$$
\int_{B} f(x) \mathbb{W}(d x)=\int_{\mathbb{R}^{d}} f(x) \mathbf{1}_{B}(x) \mathbb{W}(d x) .
$$

Theorem 26. Let $\Gamma=\mathcal{B}_{0}\left(\mathbb{R}^{d}\right)$ and $\mathbb{W}=\left\{\mathbb{W}_{A}, A \in \Gamma\right\}$ be a 1-dimensional $G$-white noise on a sublinear expectation space $(\Omega, \mathcal{H}, \hat{\mathbb{E}})$. Then $\left\{\int_{\mathbb{R}^{d}} f(x) \mathbb{W}(d x): f \in L^{2}\left(\mathbb{R}^{d}\right)\right\}$ is a G-Gaussian random field.

To prove this theorem, we need the following lemma to show that the convergence of a sequence of $G$-normally distributed random vectors is still $G$-normally distributed.

Lemma 27. Let $\left\{X_{n}\right\}_{n=1}^{\infty}$ be a sequence of $m$-dimensional G-normally distributed random variables on a sublinear expectation space $(\Omega, \mathcal{H}, \hat{\mathbb{E}})$. If for some $p \geq 1, \lim _{n \rightarrow \infty} \hat{\mathbb{E}}\left[\mid X_{n}-\right.$ $\left.\left.X\right|^{p}\right]=0$, then $X$ is also $G$-normally distributed.

Proof. As for some $p \geq 1, \lim _{n \rightarrow \infty} \hat{\mathbb{E}}\left[\left|X_{n}-X\right|^{p}\right]=0$, we can deduce that $\left\{X_{n}\right\}_{n=1}^{\infty}$ converges in distribution by

$$
\left|\mathbb{F}_{X_{n}}[\varphi]-\mathbb{F}_{X}[\varphi]\right|=\left|\hat{\mathbb{E}}\left[\varphi\left(X_{n}\right)\right]-\hat{\mathbb{E}}[\varphi(X)]\right| \leq C_{\varphi} \hat{\mathbb{E}}\left[\left|X_{n}-X\right|\right] \leq C_{\varphi} \hat{\mathbb{E}}\left[\left|X_{n}-X\right|^{p}\right]^{1 / p} \rightarrow 0
$$


as $n \rightarrow \infty$, where $C_{\varphi}$ is the Lipschitz constant of $\varphi \in C_{b . L i p}\left(\mathbb{R}^{m}\right)$. Assume that $\bar{X}_{n}$ and $\bar{X}$ are the independent copies of $X_{n}$ and $X$, respectively. Then, for each $\varphi \in C_{\text {b.Lip }}\left(\mathbb{R}^{m}\right)$, $a, b \geq 0$, we have

$$
\hat{\mathbb{E}}\left[\varphi\left(a X_{n}+b \bar{X}_{n}\right)\right]=\hat{\mathbb{E}}\left[\varphi\left(\sqrt{a^{2}+b^{2}} X_{n}\right)\right] \rightarrow \hat{\mathbb{E}}\left[\varphi\left(\sqrt{a^{2}+b^{2}} X\right)\right] \text {, as } n \rightarrow \infty .
$$

On the other hand, setting $\varphi_{n}(y)=\hat{\mathbb{E}}\left[\varphi\left(y+b X_{n}\right)\right]$ and $\bar{\varphi}(y)=\hat{\mathbb{E}}[\varphi(y+b X)]$, it is easy to show that $\varphi_{n}(y)$ is a uniform Lipschitz function with the same Lipschitz constant as $\varphi$, such that $\left|\varphi_{n}(y)-\bar{\varphi}(y)\right| \leq b C_{\varphi} \hat{\mathbb{E}}\left[\left|X-X_{n}\right|\right]$, for all $y \in \mathbb{R}^{m}$. Thus

$$
\begin{aligned}
& \left|\hat{\mathbb{E}}\left[\varphi\left(a X_{n}+b \bar{X}_{n}\right)\right]-\hat{\mathbb{E}}[\varphi(a X+b \bar{X})]\right| \\
= & \left|\hat{\mathbb{E}}\left[\varphi_{n}\left(a X_{n}\right)\right]-\hat{\mathbb{E}}[\bar{\varphi}(a X)]\right| \\
\leq & \left|\hat{\mathbb{E}}\left[\varphi_{n}\left(a X_{n}\right)\right]-\hat{\mathbb{E}}\left[\varphi_{n}(a X)\right]\right|+\hat{\mathbb{E}}\left[\left|\varphi_{n}(a X)-\bar{\varphi}(a X)\right|\right] \rightarrow 0, \quad \text { as } n \rightarrow \infty .
\end{aligned}
$$

Hence, $\hat{\mathbb{E}}[\varphi(a X+b \bar{X})] \mid=\hat{\mathbb{E}}\left[\varphi\left(\sqrt{a^{2}+b^{2}} X\right)\right]$, for each $\varphi \in C_{b . \text { Lip }}\left(\mathbb{R}^{m}\right)$, which completes the proof.

Proof of Theorem [26. For any $n \in \mathbb{N}, f_{1}, \cdots, f_{n} \in L^{2}\left(\mathbb{R}^{d}\right)$, there exists a sequence of simple functions $\left\{\left(f_{1}^{m}, \cdots, f_{n}^{m}\right), m \geq 1\right\}$ such that $\lim _{m \rightarrow \infty} f_{i}^{m}=f_{i}$ in $L^{2}\left(\mathbb{R}^{d}\right), i=1, \cdots, n$, and

$$
\left(\int_{\mathbb{R}^{d}} f_{1}(x) \mathbb{W}(d x), \cdots, \int_{\mathbb{R}^{d}} f_{n}(x) \mathbb{W}(d x)\right)=\lim _{m \rightarrow \infty}\left(\int_{\mathbb{R}^{d}} f_{1}^{m}(x) \mathbb{W}(d x), \cdots, \int_{\mathbb{R}^{d}} f_{n}^{m}(x) \mathbb{W}(d x)\right) .
$$

By Proposition 1.6 in Chapter II in Peng [15], it is easy to verify that

$$
\left(\int_{\mathbb{R}^{d}} f_{1}^{m}(x) \mathbb{W}(d x), \cdots, \int_{\mathbb{R}^{d}} f_{n}^{m}(x) \mathbb{W}(d x)\right)
$$

is $G$-normally distributed. Using Lemma 27, we deduce that

$$
\left(\int_{\mathbb{R}^{d}} f_{1}(x) \mathbb{W}(d x), \cdots, \int_{\mathbb{R}^{d}} f_{n}(x) \mathbb{W}(d x)\right)
$$

is $G$-normally distributed which completes the proof.

Example 28. Let $\left\{\mathbb{W}_{A}, A \in \mathcal{B}_{0}(\mathbb{R})\right\}$ be a 1-dimensional $G$-white noise. Define $\mathbb{B}_{t}=$ $\mathbb{W}([0, t]), t \in \mathbb{R}_{+}$, then

$$
\hat{\mathbb{E}}\left[\mathbb{B}_{t} \mathbb{B}_{s}\right]=\bar{\sigma}^{2} \lambda_{1}([0, t] \cap[0, s])=\bar{\sigma}^{2}(s \wedge t) .
$$

Different from the classical case, $\mathbb{B}_{t}$ is no longer a $G$-Brownian motion although $\mathbb{B}_{t} \stackrel{d}{=}$ $N\left(\{0\} \times\left[\underline{\sigma}^{2} t, \bar{\sigma}^{2} t\right]\right)$ for each $t \geq 0$.

4.3. The continuity of spatial $G$-white noise. For each $x \in \mathbb{R}^{d}$, let $\Gamma$ denote the set of hypercubes in $\mathbb{R}^{d}$ that contains the origin and $t$ as its two extremal vertices in the sense that

$$
\Gamma=\left\{(0 \wedge x, 0 \vee x]:=\left(0 \wedge x_{1}, 0 \vee x_{1}\right] \times \cdots \times\left(0 \wedge x_{d}, 0 \vee x_{d}\right]: \forall x=\left(x_{1}, \cdots, x_{d}\right) \in \mathbb{R}^{d}\right\}
$$

Suppose $\left(V_{\gamma}\right)_{\gamma \in \Gamma}$ is a random field in a sublinear expectation space $(\Omega, \mathcal{H}, \hat{\mathbb{E}})$. Define $V_{x}:=V_{(0 \wedge x, 0 \vee x]}, x \in \mathbb{R}^{d}$. Then $\left(V_{x}\right)_{x \in \mathbb{R}^{d}}$ is a random field indexed by $x \in \mathbb{R}^{d}$. In particular, we derive from a spatial random field $\mathbb{W}_{A}, A \in \mathcal{B}\left(\mathbb{R}^{d}\right)$. 
For the spatial white noise $\left(\mathbb{W}_{A}\right)_{A \in \mathcal{B}\left(\mathbb{R}^{d}\right)}$, the corresponding random field $\left(\mathbb{W}_{x}\right)_{x \in \mathbb{R}^{d}}$ is called $\mathbb{R}^{d}$-indexed spatial white noise. We will see in this subsection that, just like in the classical situation, this $\mathbb{R}^{d}$-indexed spatial white noise $\left(\mathbb{W}_{x}\right)_{x \in \mathbb{R}^{d}}$ has also a quasicontinuous modification in the sense of Definition 12.

We recall the following generalized Kolmogorov's continuity criterion for a random field indexed by $\mathbb{R}^{d}$ (see Denis et al. [5]).

Theorem 29. Let $p>0$ be given and $\left(V_{x}\right)_{x \in \mathbb{R}^{d}}$ be a random field such that $V_{x} \in \mathbb{L}^{p}(\Omega)$ for each $x \in \mathbb{R}^{d}$. Assume that there exist constants $c, \varepsilon>0$ such that if there exist positive constants $c, \varepsilon, p$ satisfying

$$
\hat{\mathbb{E}}\left[\left|V_{x}-V_{y}\right|^{p}\right] \leq c|x-y|^{d+\varepsilon}, \quad \text { for all } x, y \in \mathbb{R}^{d},
$$

then $\left(V_{x}\right)_{x \in[0, T]}$ has a continuous quasi-modification $\left(\tilde{V}_{x}\right)_{x \in \mathbb{R}^{d}}$ such that for each $\alpha \in$ $[0, \varepsilon / p)$,

$$
\hat{\mathbb{E}}\left[\left(\sup _{x \neq y} \frac{\left|\tilde{V}_{x}-\tilde{V}_{y}\right|}{|x-y|^{\alpha}}\right)^{p}\right]<\infty .
$$

Hence, the paths of $\tilde{V}$ are quasi-surely Hölder continuous of order $\alpha, \alpha \in[0, \varepsilon / p)$, in the sense that there exists a Borel set $N$ in with capacity 0 such that for all $\omega \in N^{c}$, the mapping $x \rightarrow \tilde{V}_{x}(\omega)$ is Holder continuous of order $\alpha$.

We have the following generalized Kolmogorov's criterion for spatial white noise $\left(\mathbb{W}_{x}\right)_{x \in \mathbb{R}^{d}}$

Theorem 30. For each $x \in \mathbb{R}^{d}$, let $\mathbb{W}_{x}:=\mathbb{W}_{(0, x]}$, be a 1 -dimensional spatial $G$-white noise on the sublinear expectation space $(\Omega, \mathcal{H}, \hat{\mathbb{E}})$. Then there exists a continuous quasimodification $\tilde{\mathbb{W}}$ of $\mathbb{W}$.

Proof. For notational simplicity, we only prove the case that $d=2$. For each $x=$ $\left(x_{1}, x_{2}\right), y=\left(y_{1}, y_{2}\right) \in \mathbb{R}^{2}$, without loss of generality, assume that $0 \leq x_{i} \leq y_{i}, i=1,2$. Then, by (4.3),

$$
\hat{\mathbb{E}}\left[\left|\mathbb{W}_{y}-\mathbb{W}_{x}\right|^{2}\right]=\bar{\sigma}^{2} \lambda_{(0, y] /(0, x]}=\bar{\sigma}^{2}\left(y_{1} y_{2}-x_{1} x_{2}\right)
$$

Thus $\mathbb{W}_{y}-\mathbb{W}_{x}$ is $G$-normally distributed and $\mathbb{W}_{y}-\mathbb{W}_{x} \stackrel{d}{=} N\left(\{0\} \times\left[\underline{\sigma}^{2}\left(y_{1} y_{2}-x_{1} x_{2}\right), \bar{\sigma}^{2}\left(y_{1} y_{2}-\right.\right.\right.$ $\left.x_{1} x_{2}\right)$ ]. It follows that

$$
\hat{\mathbb{E}}\left[\left|\mathbb{W}_{y}-\mathbb{W}_{x}\right|^{6}\right]=15 \bar{\sigma}^{6}\left|y_{1} y_{2}-x_{1} x_{2}\right|^{3}
$$

Since

$$
y_{1} y_{2}-x_{1} x_{2} \leq\left(y_{1} \vee y_{2}\right)\left(\left|y_{1}-x_{1}\right|+\left|y_{2}-x_{2}\right|\right) \leq \sqrt{2}\left(y_{1} \vee y_{2}\right)|y-x|,
$$

we have

$$
\hat{\mathbb{E}}\left[\left|\mathbb{W}_{y}-\mathbb{W}_{x}\right|^{6}\right] \leq 30 \sqrt{2} \bar{\sigma}^{6}\left(y_{1} \vee y_{2}\right)|y-x|^{3} .
$$

We then apply Theorem 29 and obtain that $\mathbb{W}$ has a continuous modification $\tilde{\mathbb{W}}$. 


\section{Spatial and temporal White noise Under G-SUblinear expectation}

5.1. Basic notions and main properties. In this section, we study a new type spatial-temporal white noise which is a random field defined on the following index set

$$
\Gamma=\left\{[s, t) \times A: 0 \leq s \leq t<\infty, A \in \mathcal{B}_{0}\left(\mathbb{R}^{d}\right)\right\} .
$$

Specifically, we have

Definition 31. A random field $\{\mathbf{W}([s, t) \times A)\}_{([s, t) \times A) \in \Gamma}$ on a sublinear expectation space $(\Omega, \mathcal{H}, \hat{\mathbb{E}})$ is called a 1 -dimensional spatial-temporal $G$-white noise if it satisfies the following conditions:

(i) For each fixed $[s, t)$, the ramdom field $\{\mathbf{W}([s, t) \times A)\}_{A \in \mathcal{B}_{0}\left(\mathbb{R}^{d}\right)}$ is a 1-dimensional spatial white-noise that has the same finite-dimensional distributions as $\left(\sqrt{t-s} \mathbb{W}_{A}\right)_{A \in \mathcal{B}_{0}\left(\mathbb{R}^{d}\right)}$

(ii) For any $r \leq s \leq t, A \in \mathcal{B}_{0}\left(\mathbb{R}^{d}\right), \mathbf{W}([r, s) \times A)+\mathbf{W}([s, t) \times A)=\mathbf{W}([r, t) \times A)$;

(iii) $\mathbf{W}([s, t) \times A)$ is independent of $\left(\mathbf{W}\left(\left[s_{1}, t_{1}\right) \times A_{1}\right), \cdots, \mathbf{W}\left(\left[s_{n}, t_{n}\right) \times A_{n}\right)\right)$, if $t_{i}<$ $s$ and $A_{i} \in \mathcal{B}_{0}\left(\mathbb{R}^{d}\right)$, for $i=1, \cdots, n$,

where $\left(\mathbb{W}_{A}\right)_{A \in \mathcal{B}_{0}\left(\mathbb{R}^{d}\right)}$ is a 1-dimensional $G$-white noise.

Remark 32. It is important to mention that $\left\{\mathbf{W}([s, t) \times A), 0 \leq s \leq t<\infty, A \in \mathcal{B}_{0}\left(\mathbb{R}^{d}\right)\right\}$ is no longer a $G$-Gaussian random field.

We now present the existence of the spatial-temporal G-white noise and the construction of the corresponding expectation and conditional expectation.

Suppose $\Omega=(\mathbb{R})^{\Gamma}$ and for each $\omega \in \Omega$, define the canonical process $\left(\mathbf{W}_{\gamma}\right)_{\gamma \in \Gamma}$ by

$$
\mathbf{W}([s, t) \times A)(\omega)=\omega([s, t) \times A), \forall 0 \leq s \leq t<\infty, A \in \mathcal{B}_{0}\left(\mathbb{R}^{d}\right) .
$$

For each $T \geq 0$, set $\mathcal{F}_{T}=\sigma\left\{\mathbf{W}([s, t) \times A), 0 \leq s \leq t \leq T, A \in \mathcal{B}\left(\mathbb{R}^{d}\right)\right\}, \mathcal{F}=\bigvee_{T \geq 0} \mathcal{F}_{T}$, and

$$
\begin{aligned}
L_{i p}\left(\mathcal{F}_{T}\right)= & \left\{\varphi\left(\mathbf{W}\left(\left[s_{1}, t_{1}\right) \times A_{1}\right), \cdots, \mathbf{W}\left(\left[s_{n}, t_{n}\right) \times A_{n}\right)\right), \quad \text { for any } n \in \mathbb{N}, s_{i} \leq t_{i} \leq T,\right. \\
& \left.i=1, \cdots, n, A_{1}, \cdots, A_{n} \in \mathcal{B}_{0}\left(\mathbb{R}^{d}\right), \varphi \in C_{l . L i p}\left(\mathbb{R}^{n}\right)\right\} .
\end{aligned}
$$

We also set $L_{i p}(\mathcal{F})=\cup_{n=1}^{\infty} L_{i p}\left(\mathcal{F}_{n}\right)$. For each $X \in L_{i p}(\mathcal{F})$, without loss of generality, we assume $X$ has the form

$$
\begin{gathered}
X=\varphi\left(\mathbf{W}\left(\left[0, t_{1}\right) \times A_{1}\right), \cdots, \mathbf{W}\left(\left[0, t_{1}\right) \times A_{m}\right), \cdots, \mathbf{W}\left(\left[t_{n-1}, t_{n}\right) \times A_{1}\right), \cdots,\right. \\
\left.\mathbf{W}\left(\left[t_{n-1}, t_{n}\right) \times A_{m}\right)\right),
\end{gathered}
$$

where $0=t_{0} \leq t_{1}<\cdots<t_{n}<\infty,\left\{A_{1}, \cdots, A_{m}\right\} \subset \mathcal{B}_{0}\left(\mathbb{R}^{d}\right)$ are mutually disjoint, and $\varphi \in C_{\text {l.Lip }}\left(\mathbb{R}^{m \times n}\right)$. Then we can define the sublinear expectation for $X$ in the following procedure.

Let $\left\{G_{A_{1}, \cdots, A_{m}}: \forall m \in \mathbb{N}, 0 \leq s<t<\infty, A_{1}, \cdots, A_{m} \in \mathcal{B}_{0}\left(\mathbb{R}^{d}\right)\right\}$ be a family of monotonic and sublinear functions defined as (4.3). By Proposition 8 , there exists a sequence of $m$-dimensional $G$-normally distributed random vectors $\left\{\xi_{1}, \cdots, \xi_{n}\right\}, \xi_{i}=$ 
$\left(\xi_{i}^{(1)}, \cdots, \xi_{i}^{(m)}\right), 1 \leq i \leq n$, on a sublinear expectation $(\tilde{\Omega}, \tilde{\mathcal{H}}, \tilde{\mathbb{E}})$ such that $\xi_{i+1}$ is independent of $\left(\xi_{1}, \cdots, \xi_{i}\right)$ for each $i=1,2, \cdots, n-1$, and

$$
G_{A_{1}, \cdots, A_{m}}(Q)=\frac{1}{2} \tilde{\mathbb{E}}\left[\left\langle Q \xi_{i}, \xi_{i}\right\rangle\right]=G\left(\sum_{i=1}^{n} q_{i i} \lambda_{A_{i}}\right), \forall Q \in \mathbb{S}(m),
$$

where $G(q)=\frac{\bar{\sigma}^{2}}{2} q^{+}-\frac{\sigma^{2}}{2} q^{-}, q \in \mathbb{R}$.

Define

$$
\begin{aligned}
& \hat{\mathbb{E}}[X]= \hat{\mathbb{E}}\left[\varphi \left(\mathbf{W}\left(\left[0, t_{1}\right) \times A_{1}\right), \cdots, \mathbf{W}\left(\left[0, t_{1}\right) \times A_{m}\right), \cdots, \mathbf{W}\left(\left[t_{n-1}, t_{n}\right) \times A_{1}\right), \cdots,\right.\right. \\
&\left.\left.\quad \mathbf{W}\left(\left[t_{n-1}, t_{n}\right) \times A_{m}\right)\right)\right] \\
&=\tilde{\mathbb{E}}\left[\varphi\left(\sqrt{t_{1}} \xi_{1}^{(1)}, \cdots, \sqrt{t_{1}} \xi_{1}^{(m)}, \cdots, \sqrt{\left(t_{n}-t_{n-1}\right)} \xi_{n}^{(1)}, \cdots, \sqrt{\left(t_{n}-t_{n-1}\right)} \xi_{n}^{(m)}\right)\right] .
\end{aligned}
$$

and the related conditional expectation of $X$ under $\mathcal{F}_{t}, t_{j} \leq t<t_{j+1}$, denoted by $\hat{\mathbb{E}}\left[X \mid \mathcal{F}_{t}\right]$, is defined by

$$
\begin{aligned}
\hat{\mathbb{E}}\left[\varphi \left(\mathbf{W}\left(\left[0, t_{1}\right) \times A_{1}\right), \cdots, \mathbf{W}\left(\left[0, t_{1}\right) \times A_{m}\right), \cdots, \mathbf{W}\left(\left[t_{n-1}, t_{n}\right) \times A_{1}\right), \cdots,\right.\right. & \\
& \left.\left.\mathbf{W}\left(\left[t_{n-1}, t_{n}\right) \times A_{m}\right)\right) \mid \mathcal{F}_{t}\right] \\
=\psi & \left(\mathbf{W}\left(\left[0, t_{1}\right) \times A_{1}\right), \cdots, \mathbf{W}\left(\left[0, t_{1}\right) \times A_{m}\right), \cdots, \mathbf{W}\left(\left[t_{j-1}, t_{j}\right) \times A_{1}\right), \cdots,\right. \\
& \left.\mathbf{W}\left(\left[t_{j-1}, t_{j}\right) \times A_{m}\right)\right) .
\end{aligned}
$$

where

$$
\begin{gathered}
\psi\left(x_{11}, \cdots, x_{j m}\right)=\tilde{\mathbb{E}}\left[\varphi \left(x_{11}, \cdots, x_{j m}, \sqrt{t_{j+1}-t_{j}} \xi_{j+1}^{(1)}, \cdots, \sqrt{t_{j+1}-t_{j}} \xi_{j+1}^{(m)}\right.\right. \\
\left.\left.\cdots, \sqrt{t_{n}-t_{n-1}} \xi_{n}^{(1)}, \cdots, \sqrt{t_{n}-t_{n-1}} \xi_{n}^{(m)}\right)\right] .
\end{gathered}
$$

It is easy to check that $\hat{\mathbb{E}}[\cdot]$ defines a sublinear expectation on $L_{i p}(\mathcal{F})$ and the canonical process $\left(\mathbf{W}_{\gamma}\right)_{\gamma \in \Gamma}$ is a 1-dimensional spatial-temporal white noise on $\left(\Omega, L_{i p}(\mathcal{F}), \hat{\mathbb{E}}\right)$.

For each $p \geq 1, T \geq 0$, we denote by $\mathbf{L}_{G}^{p}\left(\mathbf{W}_{[0, T]}\right)\left(\right.$ resp. $\left.\mathbf{L}_{G}^{p}(\mathbf{W})\right)$ the completion of $L_{i p}\left(\mathcal{F}_{T}\right)\left(\right.$ resp. $\left.L_{i p}(\mathcal{F})\right)$ under the form $\|X\|_{p}:=\left(\hat{\mathbb{E}}\left[|X|^{p}\right]\right)^{1 / p}$. The conditional expectation $\hat{\mathbb{E}}\left[\cdot \mid \mathcal{F}_{t}\right]: L_{i p}(\mathcal{F}) \rightarrow L_{i p}\left(\mathcal{F}_{t}\right)$ is a continuous mapping under $\|\cdot\|_{p}$ and can be extended continuously to the mapping $\mathbf{L}_{G}^{p}(\mathbf{W}) \rightarrow \mathbf{L}_{G}^{p}\left(\mathbf{W}_{[0, t]}\right)$ by

$$
\left|\hat{\mathbb{E}}\left[X \mid \mathcal{F}_{t}\right]-\hat{\mathbb{E}}\left[Y \mid \mathcal{F}_{t}\right]\right| \leq \hat{\mathbb{E}}\left[|X-Y| \mid \mathcal{F}_{t}\right] .
$$

It is easy to verify the conditional expectation $\hat{\mathbb{E}}\left[\cdot \mid \mathcal{F}_{t}\right]$ satisfies the following properties. We omit the proof here.

Proposition 33. The conditional expectation $\hat{\mathbb{E}}\left[\cdot \mid \mathcal{F}_{t}\right]: \mathbf{L}_{G}^{p}(\mathbf{W}) \rightarrow \mathbf{L}_{G}^{p}\left(\mathbf{W}_{[0, t]}\right)$ satisfies the following properties: for any $X, Y \in \mathbf{L}_{G}^{p}(\mathbf{W}), \eta \in \mathbf{L}_{G}^{p}\left(\mathbf{W}_{[0, t]}\right)$,

(i): $\hat{\mathbb{E}}\left[X \mid \mathcal{F}_{t}\right] \geq \hat{\mathbb{E}}\left[Y \mid \mathcal{F}_{t}\right]$ for $X \geq Y$.

(ii): $\hat{\mathbb{E}}\left[\eta \mid \mathcal{F}_{t}\right]=\eta$.

(iii): $\hat{\mathbb{E}}\left[X+Y \mid \mathcal{F}_{t}\right] \leq \hat{\mathbb{E}}\left[X \mid \mathcal{F}_{t}\right]+\hat{\mathbb{E}}\left[Y \mid \mathcal{F}_{t}\right]$.

(iv): $\hat{\mathbb{E}}\left[\eta X \mid \mathcal{F}_{t}\right]=\eta^{+} \hat{\mathbb{E}}\left[X \mid \mathcal{F}_{t}\right]+\eta^{-} \hat{\mathbb{E}}\left[-X \mid \mathcal{F}_{t}\right]$.

$(\mathrm{v}): \hat{\mathbb{E}}\left[\hat{\mathbb{E}}\left[X \mid \mathcal{F}_{t}\right] \mid \mathcal{F}_{s}\right]=\hat{\mathbb{E}}\left[X \mid \mathcal{F}_{t \wedge s}\right]$. 
Proposition 34. For each $X, Y \in \mathbf{L}_{G}^{p}\left(\mathbf{W}_{[0, T]}\right), t \leq T, \hat{\mathbb{E}}\left[Y \mid \mathcal{F}_{t}\right]=-\hat{\mathbb{E}}\left[-Y \mid \mathcal{F}_{t}\right]$. Then

$$
\hat{\mathbb{E}}\left[X+\alpha Y \mid \mathcal{F}_{t}\right]=\hat{\mathbb{E}}\left[X \mid \mathcal{F}_{t}\right]+\alpha \hat{\mathbb{E}}\left[Y \mid \mathcal{F}_{t}\right], \alpha \in \mathbb{R} .
$$

5.2. Stochastic integrals with respect to spatial-temporal white noise. In the following, we define the stochastic integral with respect to the spatial-temporal G-white noise $\mathbf{W}([s, t) \times A), 0 \leq s<t \leq T, A \in \mathcal{B}_{0}\left(\mathbb{R}^{d}\right)$.

Firstly, let $\mathbf{M}^{0}\left([0, T] \times \mathbb{R}^{d}\right)$ be the collection of simple processes with the form:

$$
f(s, x ; \omega)=\sum_{i=0}^{n-1} \sum_{j=1}^{m} X_{i j}(\omega) \mathbf{1}_{A_{j}}(x) \mathbf{1}_{\left[t_{i}, t_{i+1}\right)}(s),
$$

where $X_{i j} \in L_{i p}\left(\mathcal{F}_{t_{i}}\right), i=0, \cdots, n-1, j=1, \cdots, m, 0=t_{0}<t_{1}<\cdots<t_{n}=T$, and $\left\{A_{j}\right\}_{j=1}^{m}$ is a space partition of $\mathcal{B}_{0}\left(\mathbb{R}^{d}\right)$, namely,

$$
A_{j} \in \mathcal{B}_{0}\left(\mathbb{R}^{d}\right), \bigcup_{j=1}^{m} A_{j}=\mathbb{R}^{d}, \text { and } A_{j} \cap A_{k}=\emptyset, \quad \text { if } j \neq k, j, k=1, \cdots, m .
$$

The Bohner's integral of $f \in \mathbf{M}^{0}\left([0, T] \times \mathbb{R}^{d}\right)$ is defined by

$I_{B}(f)=\int_{\mathbb{R}^{d}} \int_{0}^{T} f(s, x) d s d x=\int_{0}^{T} \int_{\mathbb{R}^{d}} f(s, x) d x d s:=\sum_{i=0}^{n-1} \sum_{j=1}^{m} X_{i j}\left(t_{i+1}-t_{i}\right) \lambda_{A_{j}}, \quad \forall t \in[0, T]$.

It is clear that $I_{B}: \mathbf{M}^{0}\left([0, T] \times \mathbb{R}^{d}\right) \mapsto L_{i p}\left(\mathcal{F}_{T}\right)$ is a linear mapping. For a given $p \geq 1$ and $f \in \mathbf{M}^{0}\left([0, T] \times \mathbb{R}^{d}\right)$, let

$$
\|f\|_{\mathbf{M}^{p}}=\left(\hat{\mathbb{E}}\left[\int_{0}^{T} \int_{\mathbb{R}^{d}}|f(s, x)|^{p} d s d x\right]\right)^{\frac{1}{p}}=\left\{\hat{\mathbb{E}}\left[\sum_{i=0}^{n-1} \sum_{j=1}^{m} X_{i j}^{p}\left(t_{i+1}-t_{i}\right) \lambda_{A_{j}}\right]\right\}^{1 / p} .
$$

Then $\|f\|_{\mathbf{M}^{p}}$ is a Banach norm on $\mathbf{M}^{0}\left([0, T] \times \mathbb{R}^{d}\right)$ and the completion of $\mathbf{M}^{0}\left([0, T] \times \mathbb{R}^{d}\right)$ under the norm $\|\cdot\|_{\mathbf{M}^{p}}$, denoted by $\mathbf{M}_{G}^{p}\left([0, T] \times \mathbb{R}^{d}\right)$, is a Banach space.

The stochastic integral with respect to the spatial-temporal indexed white noise $\mathbf{W}$ from $\mathbf{M}^{0}\left([0, T] \times \mathbb{R}^{d}\right)$ to $L_{i p}\left(\mathcal{F}_{T}\right)$ can be defined as follows:

$$
\int_{0}^{T} \int_{\mathbb{R}^{d}} f(s, x) \mathbf{W}(d s, d x):=\sum_{i=0}^{n-1} \sum_{j=1}^{m} X_{i j} \mathbf{W}\left(\left[t_{i}, t_{i+1}\right) \times A_{j}\right) .
$$

Lemma 35. For any $0 \leq s \leq t<\infty$ and $\xi \in \mathbf{L}_{G}^{p}\left(\mathbf{W}_{[0, s]}\right)$, we have

$$
\hat{\mathbb{E}}[\xi \mathbf{W}([s, t) \times A)]=0, \quad \forall A \in \mathcal{B}_{0}(\mathbb{R}),
$$

and

$$
\hat{\mathbb{E}}\left[\xi \mathbf{W}([s, t) \times A)(\mathbf{W}([s, t) \times \bar{A})]=0, \quad \forall A, \bar{A} \in \mathcal{B}_{0}(\mathbb{R}) \text { such that } A \cap \bar{A}=\emptyset .\right.
$$

Proof. The first relation is obvious, since $\mathbf{W}([s, t) \times A)$ is independent of $\xi$ and $\hat{\mathbb{E}}[ \pm \mathbf{W}([s, t) \times$ $A)]=0$. The second relation is derived from

$$
\hat{\mathbb{E}}[ \pm \mathbf{W}([s, t) \times A)(\mathbf{W}([s, t) \times \bar{A})]=\hat{\mathbb{E}}[ \pm \mathbf{W}([s, t) \times(A \cap \bar{A}))]=0 .
$$


Using this lemma, we now provide the following lemma which allows us to extend the the domain of stochastic integral to $\mathbf{M}_{G}^{2}\left([0, T] \times \mathbb{R}^{d}\right)$ :

Lemma 36. For each $f \in \mathbf{M}^{0}\left([0, T] \times \mathbb{R}^{d}\right)$,

$$
\begin{aligned}
& \hat{\mathbb{E}}\left[\int_{0}^{T} \int_{\mathbb{R}^{d}} f(s, x) \mathbf{W}(d s, d x)\right]=0 . \\
& \hat{\mathbb{E}}\left[\left|\int_{0}^{T} \int_{\mathbb{R}^{d}} f(s, x) \mathbf{W}(d s, d x)\right|^{2}\right] \leq \bar{\sigma}^{2} \hat{\mathbb{E}}\left[\int_{0}^{T} \int_{\mathbb{R}^{d}}|f(s, x)|^{2} d s d x\right] .
\end{aligned}
$$

Proof. Suppose $f(s, x ; \omega)=\sum_{i=0}^{n-1} \sum_{j=1}^{m} X_{i j}(\omega) \mathbf{1}_{A_{j}}(x) \mathbf{1}_{\left[t_{i}, t_{i+1}\right)}(s)$, where $0 \leq t_{0}<t_{1}<\cdots<$ $t_{n} \leq T,\left\{A_{j}\right\}_{j=1}^{m} \subset \mathcal{B}_{0}\left(\mathbb{R}^{d}\right)$ is a partition of $\mathbb{R}^{d}, X_{i j} \in L_{i p}\left(\mathcal{F}_{t_{i}}\right), i=1, \cdots, n, j=$ $1, \cdots, m$. It follows from the first relation of Lemma 35, combined with Lemma 6 that

$$
\hat{\mathbb{E}}\left[\int_{0}^{T} \int_{\mathbb{R}^{d}} f(s, x) \mathbf{W}(d s, d x)\right]=\hat{\mathbb{E}}\left[\sum_{i=0}^{n-1} \sum_{j=1}^{m} X_{i j}\left(\mathbf{W}\left(\left[t_{i}, t_{i+1}\right) \times A_{j}\right)\right]=0 .\right.
$$

Hence we derive the equation (5.2). For the second one,

$$
\begin{aligned}
& \hat{\mathbb{E}} {\left[\left|\int_{0}^{T} \int_{\mathbb{R}^{d}} f(s, x) \mathbf{W}(d s, d x)\right|^{2}\right]=\hat{\mathbb{E}}\left[\left|\sum_{i=0}^{n-1} \sum_{j=1}^{m} X_{i j} \mathbf{W}\left(\left[t_{i}, t_{i+1}\right) \times A_{j}\right)\right|^{2}\right] } \\
&=\hat{\mathbb{E}}\left[\sum_{i=0}^{n-1} \sum_{j=1}^{m} \sum_{l=0}^{n-1} \sum_{k=1}^{m} X_{i j} X_{l k} \mathbf{W}\left(\left[t_{i}, t_{i+1}\right) \times A_{j}\right) \mathbf{W}\left(\left[t_{l}, t_{l+1}\right) \times A_{k}\right)\right] \\
&=\hat{\mathbb{E}}\left[\sum_{i=0}^{n-1} \sum_{j=1}^{m} \sum_{k=1}^{m} X_{i j} X_{i k} \mathbf{W}\left(\left[t_{i}, t_{i+1}\right) \times A_{j}\right) \mathbf{W}\left(\left[t_{i}, t_{i+1}\right) \times A_{k}\right)\right] .
\end{aligned}
$$

where the last equality is from the first relation of Lemma 35, combined with Lemma 6] since $A_{j} \cap A_{k}=\emptyset$ for $j \neq k$. We then apply the second relation of Lemma 35, once more combined with Lemma 6, to obtain

$$
\begin{aligned}
\hat{\mathbb{E}}\left[\left|\int_{0}^{T} \int_{\mathbb{R}^{d}} f(s, x) \mathbf{W}(d s, d x)\right|^{2}\right] & =\hat{\mathbb{E}}\left[\sum_{i=0}^{n-1} \sum_{j=1}^{m} X_{i j}^{2}\left(\mathbf{W}\left(\left[t_{i}, t_{i+1}\right) \times A_{j}\right)\right)^{2}\right] \\
& \leq \hat{\mathbb{E}}\left[\eta_{n}+\bar{\sigma}^{2} \hat{\mathbb{E}}\left[\sum_{i=0}^{n-1} \sum_{j=1}^{m} X_{i j}^{2}\left(t_{i+1}-t_{i}\right) \lambda_{A_{j}}\right]\right] \\
& =\bar{\sigma}^{2} \hat{\mathbb{E}}\left[\sum_{i=0}^{n-1} \sum_{j=1}^{m} X_{i j}^{2}\left(t_{i+1}-t_{i}\right) \lambda_{A_{j}}\right] \\
& =\bar{\sigma}^{2} \hat{\mathbb{E}}\left[\int_{0}^{T} \int_{\mathbb{R}^{d}}|f(t, x)|^{2} d x d t\right]
\end{aligned}
$$


where we denote

$$
\eta_{k}:=\sum_{i=0}^{k-1} \sum_{j=1}^{m} X_{i j}^{2}\left[\left(\mathbf{W}\left(\left[t_{i}, t_{i+1}\right) \times A_{j}\right)\right)^{2}-\bar{\sigma}^{2}\left(t_{i+1}-t_{i}\right) \lambda_{A_{j}}\right], \quad k=1, \cdots, n .
$$

Here, to obtain that $\hat{\mathbb{E}}\left[\eta_{n}\right]=0$, we take the following procedure, adopted from Peng [15.

$$
\begin{aligned}
\hat{\mathbb{E}}\left[\eta_{n}\right] & =\hat{\mathbb{E}}\left[\eta_{n-1}+\sum_{j=1}^{m} X_{n-1, j}^{2}\left[\left(\mathbf{W}\left(\left[t_{n-1}, t_{n}\right) \times A_{j}\right)\right)^{2}-\bar{\sigma}^{2}\left(t_{n}-t_{n-1}\right) \lambda_{A_{j}}\right]\right] \\
& =\hat{\mathbb{E}}\left[a+\hat{\mathbb{E}}\left[\sum_{j=1}^{m} b_{j}^{2}\left[\left(\mathbf{W}\left(\left[t_{n-1}, t_{n}\right) \times A_{j}\right)\right)^{2}-\bar{\sigma}^{2}\left(t_{n}-t_{n-1}\right) \lambda_{A_{j}}\right]\right]_{a=\eta_{n-2}, b_{j}=X_{n-1, j}}\right] \\
& =\hat{\mathbb{E}}\left[\eta_{n-2}\right]=\cdots=\hat{\mathbb{E}}\left[\eta_{1}\right]=0 .
\end{aligned}
$$

The proof is complete.

Therefore, we can continuously extend the domain $\mathbf{M}^{0}\left([0, T] \times \mathbb{R}^{d}\right)$ of the stochastic integral (5.1) to $\mathbf{M}_{G}^{2}\left([0, T] \times \mathbb{R}^{d}\right)$. Indeed, for any $f \in \mathbf{M}_{G}^{2}\left([0, T] \times \mathbb{R}^{d}\right)$, there exists a sequence of simple processes $f_{n} \in \mathbf{M}^{0}\left([0, T] \times \mathbb{R}^{d}\right)$ such that $\lim _{n \rightarrow \infty}\left\|f_{n}-f\right\|_{\mathbf{M}^{2}}=0$. By Lemma 36, we have that

$$
\left\{\int_{0}^{T} \int_{\mathbb{R}^{d}} f_{n}(s, x) \mathbf{W}(d s, d x)\right\}_{n=1}^{\infty}
$$

is a Cauchy sequence in $\mathbf{L}_{G}^{2}\left(\mathbf{W}_{[0, T]}\right)$. As $\mathbf{L}_{G}^{2}\left(\mathbf{W}_{[0, T]}\right)$ is complete, we can define

$$
\int_{0}^{T} \int_{\mathbb{R}^{d}} f(s, x) \mathbf{W}(d s, d x):=\mathbb{L}^{2}-\lim _{n \rightarrow \infty} \int_{0}^{T} \int_{\mathbb{R}^{d}} f_{n}(s, x) \mathbf{W}(d s, d x) .
$$

It is easy to check that the stochastic integral has the following properties.

Proposition 37. For each $f, g \in \mathbf{M}_{G}^{2}\left([0, T] \times \mathbb{R}^{d}\right), 0 \leq s \leq r \leq t \leq T$, we have

(1) $\int_{s}^{T} \int_{\mathbb{R}^{d}} f(s, x) \mathbf{W}(d s, d x)=\int_{s}^{r} \int_{\mathbb{R}^{d}} f(s, x) \mathbf{W}(d s, d x)+\int_{r}^{T} \int_{\mathbb{R}^{d}} f(s, x) \mathbf{W}(d s, d x)$.

(2) $\int_{s}^{T} \int_{\mathbb{R}^{d}}(\alpha f(s, x)+g(s, x)) \mathbf{W}(d s, d x)$

$=\alpha \int_{s}^{T} \int_{\mathbb{R}^{d}} f(s, x) \mathbf{W}(d s, d x)+\int_{s}^{T} \int_{\mathbb{R}^{d}} g(s, x) \mathbf{W}(d s, d x)$, if $\alpha \in \mathbf{L}_{G}^{1}\left(\mathbf{W}_{[0, s]}\right)$ is bounded.

(3) $\hat{\mathbb{E}}\left[\int_{r}^{T} \int_{\mathbb{R}^{d}} f(s, x) \mathbf{W}(d s, d x) \mid \mathcal{F}_{r}\right]=0$.

For each $f \in \mathbf{M}_{G}^{2}\left([0, T] \times \mathbb{R}^{d}\right)$, by (3) in Proposition [37, we can obtain that

$$
\left\{\int_{0}^{t} \int_{\mathbb{R}^{d}} f(r, x) \mathbf{W}(d r, d x), \quad t \in[0, T]\right\}
$$

is a martingale in the sense that

$$
\hat{\mathbb{E}}\left[\int_{0}^{t} \int_{\mathbb{R}^{d}} f(r, x) \mathbf{W}(d r, d x) \mid \mathcal{F}_{s}\right]=\int_{0}^{s} \int_{\mathbb{R}^{d}} f(r, x) \mathbf{W}(d r, d x) \quad \text { if } 0 \leq t \leq T .
$$

Furthermore, $\left\{\int_{0}^{T} \int_{\mathbb{R}^{d}} f(r, x) \mathbf{W}(d r, d x), \mathcal{F}_{t}, t \in[0, T]\right\}$ is a martingale measure. 


\section{Conclusion Remarks}

Based on a new type of G-Gaussian process introduced in Peng [17, we develop a general Gaussian random field under a sublinear expectation framework. We first construct a $G$-Gaussian random field whose finite dimensional distributions are $G$ normally distributed. A remarkable point is that, different from the classical probability theory, a $G$-Brownian motion is not a $G$-Gaussian random field. A $G$-Brownian motion is suitable to be as a temporal random field, whereas a random Gaussian field is rather suitable for describe spatial uncertainty. This new framework of $G$-Gaussian random field can be used to quantitatively and efficiently study such type of spatial uncertainties and risks.

We then define a special family of generating functions $\left\{G_{\underline{\gamma}}\right\}_{\underline{\gamma} \in \mathcal{J}_{\Gamma}}$ as (4.3) and establish the corresponding white noise of space type. Stochastic integral of functions in $L^{2}\left(\mathbb{R}^{d}\right)$ with respect to the spatial white noise has been established and the family of stochastic integrals is still a $G$-Gaussian random field.

Furthermore, we focus on the spatial-temporal $G$-white noise on the sublinear expectation space. Applying the similar method of $G$-Itô's integral, we develop the stochastic calculus with respect to the spatial-temporal white noise on $\mathbf{M}_{G}^{2}\left([0, T] \times \mathbb{R}^{d}\right)$.

$G$-Gaussian random field and $G$-white noise theory can be widely used to study the uncertain models in many physical fields, such as statistical physics and quantum theory.

\section{ApPEndix}

7.1. Upper expectation and the corresponding capacity. Let $\Gamma$ be defined as (4.6). In this section, unless otherwise mentioned, we always denote by $\Omega=C_{0}(\Gamma, \mathbb{R})$, the space of all $\mathbb{R}$-valued continuous functions $\left(\omega_{\gamma}\right)_{\gamma \in \Gamma}$, with $\omega_{0}=0$, equipped with the distance

$$
\rho\left(\omega^{(1)}, \omega^{(2)}\right):=\sum_{i=1}^{\infty} 2^{-i}\left[\left(\max _{\gamma \subset \mathfrak{B}^{d}(0 ; i)}\left|\omega_{\gamma}^{(1)}-\omega_{\gamma}^{(2)}\right|\right) \wedge 1\right], \forall \omega^{(1)}, \omega^{(2)} \in \Omega,
$$

where $\mathfrak{B}^{d}(0 ; i)=\left\{x \in \mathbb{R}^{d}:|x| \leq i\right\}$. Let $\bar{\Omega}=\mathbb{R}^{\Gamma}$ denote the space of all $\mathbb{R}$-valued functions $\left(\bar{\omega}_{\gamma}\right)_{\gamma \in \Gamma \text {. }}$.

We also denote by $\mathcal{B}(\Omega)$, the $\sigma$-algebra generated by all open sets and let $\mathcal{B}(\bar{\Omega})$ be the $\sigma$-algebra generated by all finite dimensional cylinder sets. The corresponding canonical process is $\mathbb{W}_{\gamma}(\omega)=\omega_{\gamma}$ (respectively, $\left.\overline{\mathbb{W}}_{\gamma}(\bar{\omega})=\bar{\omega}_{\gamma}\right), \gamma \in \Gamma$ for $\omega \in \Omega$ (respectively, $\bar{\omega} \in \bar{\Omega})$.

The spaces of Lipschitz cylinder functions on $\Omega$ and $\bar{\Omega}$ are denoted respectively by

$$
\begin{aligned}
& L_{i p}(\Omega):=\left\{\varphi\left(\mathbb{W}_{\gamma_{1}}, \mathbb{W}_{\gamma_{2}}, \cdots, \mathbb{W}_{\gamma_{n}}\right): \forall n \geq 1, \gamma_{1}, \cdots, \gamma_{n} \in \Gamma, \forall \varphi \in C_{\text {l.Lip }}\left(\mathbb{R}^{n}\right)\right\}, \\
& L_{i p}(\bar{\Omega}):=\left\{\varphi\left(\overline{\mathbb{W}}_{\gamma_{1}}, \overline{\mathbb{W}}_{\gamma_{2}}, \cdots, \overline{\mathbb{W}}_{\gamma_{n}}\right): \forall n \geq 1, \gamma_{1}, \cdots, \gamma_{n} \in \Gamma, \forall \varphi \in C_{\text {l.Lip }}\left(\mathbb{R}^{n}\right)\right\} .
\end{aligned}
$$

Let $\left\{G_{\underline{\gamma}}(\cdot), \underline{\gamma} \in \mathcal{J}_{\Gamma}\right\}$ be a family of continuous monotone and sublinear functions given by (4.3). Following Section 4, we can construct the corresponding sublinear expectation $\overline{\mathbb{E}}$ on $\left(\bar{\Omega}, L_{i p}(\bar{\Omega})\right)$. Due to the natural correspondence of $L_{i p}(\bar{\Omega})$ and $L_{i p}(\Omega)$, 
we also construct a sublinear expectation $\hat{\mathbb{E}}$ on $\left(\Omega, L_{i p}(\Omega)\right)$ such that $\left(\mathbb{W}_{\gamma}(\omega)\right)_{\gamma \in \Gamma}$ is a $G$-white noise.

We want to construct a weakly compact family of ( $\sigma$-additive) probability measures $\mathcal{P}$ on $(\Omega, \mathcal{B}(\Omega))$ such that the sublinear expectation can be represented as an upper expectation, namely,

$$
\hat{\mathbb{E}}[\cdot]=\max _{P \in \mathcal{P}} E_{P}[\cdot] .
$$

The following lemmas are variations of Lemma 3.3 and 3.4 in Chapter I of Peng [15].

We denote $\mathcal{J}_{\Gamma}:=\left\{\underline{\gamma}=\left(\gamma_{1}, \ldots, \gamma_{m}\right): \forall m \in \mathbb{N}, \gamma_{i} \in \Gamma\right\}$.

Lemma 38. Let $\underline{\gamma}=\left(\gamma_{1}, \cdots, \gamma_{m}\right) \in \mathcal{J}_{\Gamma}$ and $\left\{\varphi_{n}\right\}_{n=1}^{\infty} \subset C_{\text {l.Lip }}\left(\mathbb{R}^{m}\right)$ satisfy $\varphi_{n} \downarrow 0$ as $n \rightarrow \infty$. Then $\overline{\mathbb{E}}\left[\bar{\varphi}_{n}\left(\overline{\mathbb{W}}_{\gamma_{1}}, \overline{\mathbb{W}}_{\gamma_{2}}, \cdots, \overline{\mathbb{W}}_{\gamma_{m}}\right)\right] \downarrow 0$.

Lemma 39. Let $E$ be a finitely additive linear expectation dominated by $\overline{\mathbb{E}}$ on $L_{i p}(\bar{\Omega})$. Then there exists a unique probability measure $P$ on $(\bar{\Omega}, \mathcal{B}(\bar{\Omega}))$ such that $E[X]=E_{P}[X]$ for each $X \in L_{i p}(\bar{\Omega})$.

Proof. For any fixed $\underline{\gamma}=\left(\gamma_{1}, \ldots, \gamma_{m}\right) \in \mathcal{J}_{\Gamma}$, by Lemma 38 , for each sequence $\left\{\varphi_{n}\right\}_{n=1}^{\infty} \subset$ $C_{\text {l.Lip }}\left(\mathbb{R}^{m}\right)$ satisfying $\varphi_{n} \downarrow 0$, we have $\overline{\mathbb{E}}\left[\varphi_{n}\left(\overline{\mathbb{W}}_{\gamma_{1}}, \overline{\mathbb{W}}_{\gamma_{2}}, \cdots, \overline{\mathbb{W}}_{\gamma_{m}}\right)\right] \downarrow 0$. By DaniellStone's theorem (see Appendix B in Peng [15]), there exists a unique probability measure $P_{\underline{\gamma}}$ on $\left(\mathbb{R}^{m}, \mathcal{B}\left(\mathbb{R}^{m}\right)\right)$ such that $E_{P_{\underline{\gamma}}}[\varphi]=\overline{\mathbb{E}}\left[\varphi\left(\overline{\mathbb{W}}_{\gamma_{1}}, \overline{\mathbb{W}}_{\gamma_{2}}, \cdots, \overline{\mathbb{W}}_{\gamma_{m}}\right)\right]$ for each $\varphi \in C_{l . L i p}\left(\overline{\mathbb{R}}^{m}\right)$. Thus we get a family of finite dimensional distributions $\left\{P_{\underline{\gamma}}: \underline{\gamma} \in \mathcal{J}_{\Gamma}\right\}$. It is easy to check that $\left\{P_{\underline{\gamma}}: \underline{\gamma} \in \mathcal{J}_{\Gamma}\right\}$ is a consistent family. Then by Kolmogorov's consistent extension theorem, there exists a probability measure $P$ on $(\bar{\Omega}, \mathcal{B}(\bar{\Omega}))$ such that $\left\{P_{\underline{\gamma}}: \underline{\gamma} \in \mathcal{J}_{\Gamma}\right\}$ is the finite dimensional distributions of $P$. We now prove the uniqueness. Assume that there exists another probability measure $\bar{P}$ satisfying the condition. By Daniell-Stone's theorem, $P$ and $\bar{P}$ have the same finite-dimensional distributions, hence by the monotone class theorem, $P=\bar{P}$. The proof is complete.

Lemma 40. There exists a family of probability measures $\mathcal{P}_{e}$ on $(\bar{\Omega}, \mathcal{B}(\bar{\Omega}))$ such that

$$
\overline{\mathbb{E}}[X]=\max _{P \in \mathcal{P}_{e}} E_{P}[X], \quad \text { for } X \in L_{i p}(\bar{\Omega}) .
$$

Proof. By the representation theorem of the sublinear expectation and Lemma 39, it is easy to get the result.

For this $\mathcal{P}_{e}$, we define the associated capacity:

$$
\tilde{c}(A):=\sup _{P \in \mathcal{P}_{e}} P(A), \quad A \in \mathcal{B}(\bar{\Omega}),
$$

and the upper expectation for each $\mathcal{B}(\bar{\Omega})$-measurable real function $X$ which makes the following definition meaningful:

$$
\tilde{\mathbb{E}}[X]:=\sup _{P \in \mathcal{P}_{e}} E_{P}[X]
$$

By Theorem 30, there exists a continuous modification $\tilde{\mathbb{W}}$ of $\overline{\mathbb{W}}$ in the sense that $\tilde{c}\left(\left\{\tilde{\mathbb{W}}_{\gamma} \neq \overline{\mathbb{W}}_{\gamma}\right\}\right)=0$, and

$\tilde{\mathbb{E}}\left[\left|\tilde{\mathbb{W}}_{\gamma_{1}}-\tilde{\mathbb{W}}_{\gamma_{2}}\right|^{2 d+2}\right]=\tilde{\mathbb{E}}\left[\left|\overline{\mathbb{W}}_{\gamma_{1}}-\overline{\mathbb{W}}_{\gamma_{2}}\right|^{2 d+2}\right]=\overline{\mathbb{E}}\left[\left|\overline{\mathbb{W}}_{\gamma_{1}}-\overline{\mathbb{W}}_{\gamma_{2}}\right|^{2 d+2}\right]=L\left|\gamma_{1}-\gamma_{2}\right|^{d+1}, \forall \gamma_{1}, \gamma_{2} \in \Gamma$, 
where $L$ is a constant depending only on $\bar{E}$.

For any $P \in \mathcal{P}_{e}$, let $P \circ \tilde{\mathbb{W}}^{-1}$ denote the probability measure on $(\Omega, \mathcal{B}(\Omega))$ induced by $\tilde{\mathbb{W}}$ with respect to $P$. We denote $\mathcal{P}_{1}=\left\{P \circ \tilde{\mathbb{W}}^{-1}: P \in \mathcal{P}_{e}\right\}$. Applying the well-known criterion for tightness of Kolmogorov-Chentsov's type expressed in terms of moments (see Appendix B in Peng [15]), we conclude that $\mathcal{P}_{1}$ is tight. We denote by $\mathcal{P}=\overline{\mathcal{P}}_{1}$ the closure of $\mathcal{P}_{1}$ under the topology of weak convergence, then $\mathcal{P}$ is weakly compact.

Now, we give the representation of the sublinear expectation.

Theorem 41. Let $\left\{G_{\underline{\gamma}}(\cdot), \underline{\gamma} \in \mathcal{J}_{\Gamma}\right\}$ be a family of continuous monotone and sublinear functions given by $(4.3)$ and $\hat{\mathbb{E}}$ be the corresponding sublinear expectation on $\left(\Omega, L_{i p}(\Omega)\right)$. Then there exists a weakly compact family of probability measures $\mathcal{P}$ on $(\Omega, \mathcal{B}(\Omega)$ ) such that

$$
\hat{\mathbb{E}}[X]=\max _{P \in \mathcal{P}} E_{P}[X] \text { for } X \in L_{i p}(\Omega) .
$$

Proof. By Lemma 40, we have

$$
\hat{\mathbb{E}}[X]=\max _{P \in \mathcal{P}_{1}} E_{P}[X] \text { for } X \in L_{i p}(\Omega) .
$$

For any $X \in L_{i p}(\Omega)$, by Lemma 38, we get $\hat{\mathbb{E}}[|X-(X \wedge N) \vee(-N)|] \downarrow 0$ as $N \rightarrow \infty$. Noting that $\mathcal{P}=\overline{\mathcal{P}}_{1}$, by the definition of weak convergence, we thus have the result.

\section{REFERENCES}

[1] Artzner, Ph., Delbaen, F., Eber, J. M., and Heath, D., Coherent Measures of Risk. Mathematical Finance, 9(1999), no. 3, 203-228.

[2] Dalang, Robert C., Extending the martingale measure stochastic integral with applications to spatially homogeneous s.p.d.e.'s. Electron. J. Probab. 4(1999), no. 6, 29 pp.

[3] Da Prato, G., and Zabczyk, J., Stochastic equations in infinite dimensions, Cambridge University Press, Cambridge, 1992.

[4] Delbaen, F. (2002) Coherent Risk Measures (Lectures given at the Cattedra Galileiana at the Scuola Normale di Pisa, March 2000), Published by the Scuola Normale di Pisa.

[5] Denis, L., Hu, M., Peng, S., Function spaces and capacity related to a sublinear expectation: application to G-Brownian motion paths, Potential Analysis 34(2): 139-161, 2011.

[6] Föllmer, H., Spatial risk measures and their local specification: the locally law-invariant case. Stat. Risk Model. 31 (2014), no. 1, 79-101.

[7] Föllmer, H., and Klüppelberg C., Spatial risk measures: local specification and boundary risk. Stochastic analysis and applications 2014, 307-326, Springer Proc. Math. Stat., 100, Springer, Cham, 2014.

[8] Föllmer, H., and Schied, A., Convex measures of risk and trading constraints. Finance Stoch. 6 (2002), no. 4, 429-447.

[9] Huber, P. J., Robust Statistics. John Wiley \& Sons, Inc., New York, 1981.

[10] Khoshnevisan, D., Analysis of stochastic partial differential equations. CBMS Regional Conference Series in Mathematics, 119. American Mathematical Society,Providence, RI, 2014.

[11] Khoshnevisan, D., Multiparameter processes: an introduction to random fields. Springer Monographs in Mathematics. Springer-Verlag, New York, 2002.

[12] Peng, S., G-expectation, G-Brownian motion and related stochastic calculus of Itô's type, Stochastic analysis and applications, 541-567, Abel Symposia 2, Springer, Berlin, 2007.

[13] Peng, S., Multi-dimensional G-Brownian motion and related stochastic calculus under Gexpectation, Stochastic Processes and their Applications 118(12): 2223-2253, 2008. 
[14] Peng, S., A new central limit theorem under sublinear expectations, in arXiv:0803.2656, 2008.

[15] Peng, S., Nonlinear expectations and stochastic calculus under uncertainty, arXiv:1002.4546v1, 2010.

[16] Peng, S., Tightness, weak compactness of nonlinear expectations and application to CLT, arXiv:1006.2541v1, 2010.

[17] Peng, S., G-Gaussian Processes under sublinear expectations and $q$-Brownian motion in quantum mechanics, arXiv:1105.1055v1, 2011.

[18] Walley, P., Statistical Reasoning with Imprecise Probabilities. Chapman and Hall, London, New York, 1991.

[19] Walsh, J. B., An introduction to stochastic partial differential equations. École d'été de Prob. de St-Flour XIV, 1984, 265-439, Lect. Notes in Math., 1180, Springer, Berlin, 1986.

School of Mathematics Shandong University, 250100, Jinan, this Research is SupPORTED BY NSF OF ChINA No. L1624032

E-mail address: Ji Xiaojun: jixiaojun@mail.sdu.edu.cn, Peng Shige: peng@sdu.edu.cn 